\title{
Nonlinear Semigroups Built on Generating Families and their Lipschitz Sets
}

\author{
Jonas Blessing ${ }^{1} \cdot$ Michael Kupper $^{1}$ iD
}

Received: 21 December 2020 / Accepted: 5 January 2022 / Published online: 27 January 2022

(C) The Author(s) 2022

\begin{abstract}
Under suitable conditions on a family $(I(t))_{t>0}$ of Lipschitz mappings on a complete metric space, we show that, up to a subsequence, the strong limit $S(t):=\lim _{n \rightarrow \infty}\left(I\left(t 2^{-n}\right)\right)^{2^{n}}$ exists for all dyadic time points $t$, and extends to a strongly continuous semigroup $(S(t))_{t \geq 0}$. The common idea in the present approach is to find conditions on the generating family $(I(t))_{t \geq 0}$, which can be transferred to the semigroup. The construction relies on the Lipschitz set, which is invariant under iterations and allows to preserve Lipschitz continuity to the limit. Moreover, we provide a verifiable condition which ensures that the infinitesimal generator of the semigroup is given by $\lim _{h \downarrow 0} \frac{I(h) x-x}{h}$, whenever this limit exists. The results are illustrated with several examples of nonlinear semigroups such as robustifications and perturbations of linear semigroups.
\end{abstract}

Keywords Nonlinear semigroup - Infinitesimal generator · Lipschitz set . Chernoff approximation

Mathematics Subject Classification (2010) Primary 47H20 • 47J25 • Secondary 47J35 . $35 \mathrm{~B} 20 \cdot 35 \mathrm{~K} 55$

\section{Introduction}

Let $X$ be a complete metric space and $(I(t))_{t \geq 0}$ a family of Lipschitz continuous mappings $I(t): X \rightarrow X$. We are interested in the question whether one can construct an associated semigroup $(S(t))_{t \geq 0}$, which satisfies

$$
S(t) x=\lim _{n \rightarrow \infty}\left(I\left(2^{-n} t\right)\right)^{2^{n}} x .
$$

We thank Robert Denk, Stephan Eckstein, Karsten Herth, Markus Kunze, Max Nendel, Reinhard Racke and Liming Yin for helpful comments and discussions. Furthermore, we thank an anonymous referee for valuable comments and feedback on an earlier version of the paper.

Jonas Blessing

jonas.blessing@uni-konstanz.de

Michael Kupper

kupper@uni-konstanz.de

1 Department of Mathematics and Statistics, University of Konstanz, Konstanz, Germany 
Formulas of this type are called Chernoff approximation. In his monograph [8], Chernoff generalized his previous work [7] and the results by Trotter [33, 34]: under additional stability conditions on the approximating sequence $\left(I\left(2^{-n} t\right)^{2^{n}} x\right)_{n \in \mathbb{N}}$ and the assumption that $(I(t))_{t \geq 0}$ is strongly continuous, it was shown in [8, Theorem 2.5.3] that the family $(S(t))_{t>0}$ is a strongly continuous semigroup of Lipschitz continuous mappings.

In this article, we provide a detailed study how a modified version of the Chernoff approximation can be used for the construction of nonlinear semigroups. The key idea is to find properties of $(I(t))_{t \geq 0}$, which are preserved during the iteration, and can therefore be transferred to the semigroup $(S(t))_{t \geq 0}$. Typically, the family $(I(t))_{t \geq 0}$ has a representation, which allows for explicit verification of these properties. Compared to formula Eq. (1.1), we take the limit only along a convergent subsequence, i.e.,

$$
S(t) x=\lim _{l \rightarrow \infty} I\left(2^{-n_{l}} t\right)^{2^{n_{l}} x} .
$$

Clearly, under suitable compactness and separability assumptions, we can choose a diagonal sequence for all $(x, t)$ in a countable dense subset $\mathcal{D} \times \mathcal{T}$ of $X \times \mathbb{R}_{+}$. Hence, we have to extend the mapping $\mathcal{D} \times \mathcal{T} \rightarrow X,(x, t) \mapsto S(t) x$ to the closure $X \times \mathbb{R}_{+}$. To do so, we need sufficient Lipschitz continuity of $(I(t))_{t \geq 0}$ in the variables $(x, t) \in \mathcal{D} \times \mathcal{T}$, which is preserved during the construction. In several examples, the boundedness and Lipschitz assumptions on the family $(I(t))_{t \geq 0}$ are easily verified. The verification of the relative compactness of the sequence $\left(I\left(2^{-n} t\right)^{2^{n}} x\right)_{n \in \mathbb{N}}$ is more involved and depends on the choice of the space $X$. The described construction leads to a strongly continuous semigroup $(S(t))_{t \geq 0}$ of locally Lipschitz continuous operators $S(t): X \rightarrow X$.

Another focus of this work is the connection between the local behaviour of $(I(t))_{t \geq 0}$ and $(S(t))_{t \geq 0}$. Let $X$ be a Banach space. If $(I(t))_{t \geq 0}$ is a family of linear contractions, it was shown in [8, Theorem 3.7] that the infinitesimal generator of $(S(t))_{t \geq 0}$ is an extension of the derivative $I^{\prime}(0)$. In this spirit, in Theorem 4.3, we provide an abstract technical condition, which ensures that

$$
\lim _{t \downarrow 0}\left\|\frac{I(t) x-x}{t}-y\right\|=0 \quad \text { implies } \quad \lim _{t \downarrow 0}\left\|\frac{S(t) x-x}{t}-y\right\|=0 .
$$

For instance, this result always holds, if $(I(t))_{t \geq 0}$ consists of convex monotone operators. We also discuss whether $(S(t))_{t \geq 0}$ represents the unique solution to the abstract Cauchy problem

$$
\partial_{t} u(t)=A u(t) \quad \text { for all } t \geq 0, \quad u(0)=x,
$$

where $A$ denotes the generator of $(S(t))_{t \geq 0}$ and $x \in D(A)$. Unlike to the theory of linear semigroups, this is not immediately clear and depends strongly on additional properties of $X$. In particular, one has to verify $S(t): D(A) \rightarrow D(A)$ for all $t \geq 0$, which is in general wrong, see [13]. In contrast, the invariance of the symmetric Lipschitz set [13] does not depend on $X$, but rather on convexity and monotonicity of $(S(t))_{t \geq 0}$. We provide sufficient conditions on $(S(t))_{t \geq 0}$ for the invariance and investigate how these conditions can be derived from $(I(t))_{t \geq 0}$. Furthermore, the symmetric Lipschitz set can be completely 
described by $(I(t))_{t \geq 0}$, and therefore explicitly determined in examples, see Section 6.1. Finally, we want to emphasise that the results on the generator and the symmetric Lipschitz set do not require norm convergence of the approximating sequence as in Eq. (1.2).

The present approach is inspired by the Nisio semigroup [26], where the sequence $\left(I\left(2^{-n} t\right)^{2^{n}} x\right)_{n \in \mathbb{N}}$ is non-decreasing. In this case, the limit in Eq. (1.2) does not depend on the choice of the convergent subsequence, i.e., Eqs. (1.1) and (1.2) are equivalent, see Section 2.2. The Nisio semigroup is obtained from the family $I(t) x:=\sup _{\lambda \in \Lambda} S_{\lambda}(t) x$, where $\left(S_{\lambda}\right)_{\lambda \in \Lambda}$ is a family of monotone linear semigroups. In case that each $\left(S_{\lambda}(t)\right)_{t \geq 0}$ is the semigroup of a Markov process, the respective Nisio semigroup corresponds to a stochastic process under parameter uncertainty, see [12, 24]. Typical examples include Brownian motions with drift or volatility uncertainty, see [9, 29, 30], and Lévy processes with uncertainty in the Lévy triplet, see [18, 19, 22, 25]. Compared to the Nisio semigroup, the construction based on the norm convergence as in Eq. (1.2) does not rely at all on monotonicity and we do not require $(I(t))_{t \geq 0}$ to be the supremum over a family of monotone linear semigroups. For instance, this is illustrated in Section 6.5 by means of reaction-diffusion equations, where the operators $I(t)$ are neither convex nor monotone.

In the literature, the Chernoff approximation is mainly used as a tool for finding approximative representations of semigroups or solutions of evolution equations, for which the existence is already established. In many applications the goal is to find an approximation of the form Eq. (1.1) to give explicit representations of semigroups, see, e.g., [6, 27, 31]. For a survey of Chernoff approximations of operator semigroups we refer to [5], see also [17] for an overview on different kinds of approximations. A classical approach to nonlinear PDEs and the respective nonlinear semigroups is based on the theory of maximal monotone or maccretive operators, see [1, 2, 4, 16, 20]. For HJB-type equations, as outlined in [13], it is rather delicate to verify the m-accreditivity. Furthermore, the theory of backward stochastic differential equations provides a powerful tool for the representation of second order quasi-linear equations, see, e.g., [11, 15], and [21, 32] for fully nonlinear equations.

The paper is organized as follows. In Section 2, we introduce the basic assumptions and state the main result on the existence of nonlinear semigroups. In Section 3, we investigate how to verify the imposed compactness assumption for certain spaces of continuous functions. In Section 4, we prove that the generator of $(S(t))_{t \geq 0}$ is an extension of the derivative $I^{\prime}(0)$. In Section 5, we study the symmetric Lipschitz set. Finally, in Section 6, the main results are illustrated with several examples.

\section{Construction of Nonlinear Semigroups}

Let $(X, d)$ be a complete metric space. We denote by $B(x, r):=\{y \in X: d(x, y) \leq r\}$ the closed ball with radius $r \geq 0$ around $x \in X$, and by $\mathbb{R}_{+}:=\{x \in \mathbb{R}: x \geq 0\}$ the positive real numbers including zero. Let $(I(t))_{t \geq 0}$ be a family of operators $I(t): X \rightarrow X$ satisfying the following boundedness and Lipschitz conditions.

Assumption 2.1 The family $(I(t))_{t \geq 0}$ satisfies the following properties:

(i) $I(0)=\mathrm{id}_{X}$.

(ii) There exists $x_{0} \in X$ such that

$$
I(t): B\left(x_{0}, r\right) \rightarrow B\left(x_{0}, \alpha(r, t)\right) \text { for all } r, t \geq 0,
$$


where $\alpha: \mathbb{R}_{+} \times \mathbb{R}_{+} \rightarrow \mathbb{R}_{+}$is a function, which is non-decreasing in the second argument, and satisfies

$$
\alpha(\alpha(r, s), t) \leq \alpha(r, s+t) \text { for all } r, s, t \geq 0 .
$$

(iii) For every $r \geq 0$, there exists $\omega_{r} \geq 0$ such that

$$
d(I(t) x, I(t) y) \leq e^{t \omega_{r}} d(x, y) \text { for all } t \in[0,1] \text { and } x, y \in B\left(x_{0}, r\right) .
$$

Remark 2.2 (i) It holds $\alpha(r, t) \geq r$ for all $r, t \geq 0$, because the non-decreasingness of $\alpha$ in the second argument and $I(0)=\mathrm{id}_{X}$ imply $\alpha(r, t) \geq \alpha(r, 0) \geq r$. Furthermore, we assume w.l.o.g. that the mapping $\mathbb{R}_{+} \rightarrow \mathbb{R}_{+}, r \mapsto w_{r}$ is non-decreasing.

(ii) Let $(I(t))_{t \geq 0}$ be a strongly continuous semigroup of bounded linear operators on a Banach space $(X,\|\cdot\|)$. Then, there exist $M \geq 1$ and $\omega \in \mathbb{R}$ such that $\|I(t) x\| \leq$ $M e^{\omega t}\|x\|$ for all $t \geq 0$ and $x \in X$, see [28]. If $(I(t))_{t \geq 0}$ is quasi-contractive, i.e. $M=1$, Assumption 2.1(iii) is satisfied. In the nonlinear case, the exponent $\omega_{r}$ might depend on $r$. Since we are only interested in the short time behaviour of $(I(t))_{t \geq 0}$, we do not require this property for $t>1$.

Definition 2.3 Let $(J(t))_{t \geq 0}$ be a family of operators $J(t): X \rightarrow X$. The Lipschitz set $\mathcal{L}^{J}$ consists of all $x \in X$, for which there exist $t_{0}>0$ and $c \geq 0$ with

$$
d(J(t) x, x) \leq c t \quad \text { for all } t \in\left[0, t_{0}\right] .
$$

For convex semigroups, the Lipschitz set was introduced in [13]. The Lipschitz set allows us to establish strong continuity of the semigroup $S$, and will be used in Section 6.1 to prove a regularity result.

Let $\mathcal{T}:=\left\{k 2^{-n}: k, n \in \mathbb{N}_{0}\right\}$ be the set of all positive dyadic numbers, which is countable and dense in $\mathbb{R}_{+}$. We define the partitions $\pi_{n}^{t}:=\left\{k 2^{-n} \wedge t: k \in \mathbb{N}_{0}\right\}$ and the iterated operators $I\left(\pi_{n}^{t}\right):=I\left(2^{-n}\right)^{2^{n} t}$ for all $t \in \mathcal{T}$ and $n \in \mathbb{N}$ with $2^{n} t \in \mathbb{N}$. The verification of the following compactness assumption will be discussed in Section 3 for certain spaces of continuous functions.

Assumption 2.4 There exists a countable set $\mathcal{D} \subset \mathcal{L}^{I}$, which is dense in $X$. Furthermore, the sequence $\left(I\left(\pi_{n}^{t}\right) x\right)_{n \in \mathbb{N}}$ is relatively compact in $X$ for all $(x, t) \in \mathcal{D} \times \mathcal{T}$.

The previous assumption implies the existence of a subsequence $\left(n_{l}\right)_{l \in \mathbb{N}} \subset \mathbb{N}$ such that we can define $S(t) x:=\lim _{l \rightarrow \infty} I\left(\pi_{n_{l}}^{t}\right) x$ for all $(x, t) \in \mathcal{D} \times \mathcal{T}$. Then, since the Lipschitz continuity of $(I(t))_{t \geq 0}$ in the variables $(x, t) \in \mathcal{D} \times \mathcal{T}$ from Assumption 2.1 and Definition 2.3 is preserved during the iteration and in the limit, we can extend the mapping $\mathcal{D} \times \mathcal{T} \rightarrow X,(x, t) \mapsto S(t) x$ to the closure $\overline{\mathcal{D} \times \mathcal{T}}=X \times \mathbb{R}_{+}$. This leads to the first main result.

Theorem 2.5 Suppose that $(I(t))_{t \geq 0}$ satisfies Assumptions 2.1 and 2.4. Then, there exists a family $(S(t))_{t \geq 0}$ of operators $S(t): X \rightarrow X$ with the following properties:

(i) There exists a subsequence $\left(n_{l}\right)_{l \in \mathbb{N}} \subset \mathbb{N}$ such that

$$
S(t) x=\lim _{l \rightarrow \infty} I\left(\pi_{n_{l}}^{t}\right) x \quad \text { for all }(x, t) \in X \times \mathcal{T} .
$$

(ii) The family $(S(t))_{t \geq 0}$ forms a semigroup, i.e.,

$$
S(0)=\operatorname{id}_{X} \quad \text { and } \quad S(s+t)=S(s) S(t) \quad \text { for all } s, t \geq 0 .
$$


(iii) For every $r, t \geq 0$,

$$
\begin{aligned}
d\left(x_{0}, S(t) x\right) & \leq \alpha(r, t) \quad \text { for all } x \in B\left(x_{0}, r\right), \\
d(S(t) x, S(t) y) & \leq e^{t \omega_{\alpha(r, t)}} d(x, y) \quad \text { for all } x, y \in B\left(x_{0}, r\right) .
\end{aligned}
$$

(iv) The semigroup $(S(t))_{t \geq 0}$ is strongly continuous, i.e., the mapping

$$
\mathbb{R}_{+} \rightarrow X, t \mapsto S(t) x
$$

is continuous for all $x \in X$.

(v) For every $r \geq 0$ and $x \in B\left(x_{0}, r\right) \cap \mathcal{L}^{I}$, there exists $c \geq 0$ such that

$$
d(S(s) x, S(t) x) \leq c e^{T \omega_{\alpha(r, T)}}|s-t| \quad \text { for all } T \geq 0 \text { and } s, t \in[0, T] .
$$

We call $(I(t))_{t \geq 0}$ a generating family of the semigroup $(S(t))_{t \geq 0}$, and $(S(t))_{t \geq 0}$ an associated semigroup to the family $(I(t))_{t \geq 0}$.

Corollary 2.6 It holds $\mathcal{L}^{I} \subset \mathcal{L}^{S}$ and $S(t): \mathcal{L}^{S} \rightarrow \mathcal{L}^{S}$ for all $t \geq 0$.

Proof The inclusion $\mathcal{L}^{I} \subset \mathcal{L}^{S}$ follows from Theorem $2.5(\mathrm{v})$ and $S(0)=\operatorname{id}_{X}$. Fix $x \in \mathcal{L}^{S}$ and choose $t_{0}>0$ and $c \geq 0$ such that

$$
d(S(t) x, x) \leq c t \quad \text { for all } t \in\left[0, t_{0}\right] .
$$

We use Theorem 2.5(iii), inequality (2.2) and inequality (2.1) to conclude that

$$
\begin{aligned}
d(S(s) S(t) x, S(t) x) & =d(S(t) S(s) x, S(t) x) \leq e^{t \omega_{\alpha(\alpha(r, s), t)}} d(S(s) x, x) \\
& \leq c e^{t \omega_{\alpha(r, s+t)}} \quad \text { for all } t \geq 0 \text { and } s \in\left[0, t_{0}\right] .
\end{aligned}
$$

\subsection{Proof of Theorem 2.5}

In the sequel, we establish a series of lemmas, which prove Theorem 2.5. We will always suppose that Assumptions 2.1 and 2.4 hold.

Lemma 2.7 For every $r \geq 0, t \in \mathcal{T}$ and $n \in \mathbb{N}$ with $2^{n} t \in \mathbb{N}$,

$$
\begin{aligned}
d\left(x_{0}, I\left(\pi_{n}^{t}\right) x\right) & \leq \alpha(r, t) \quad \text { for all } x \in B\left(x_{0}, r\right), \\
d\left(I\left(\pi_{n}^{t}\right) x, I\left(\pi_{n}^{t}\right) y\right) & \leq e^{t \omega_{\alpha(r, t)}} d(x, y) \quad \text { for all } x, y \in B\left(x_{0}, r\right) .
\end{aligned}
$$

Proof Fix $n \in \mathbb{N}$. First, we show by induction that

$$
I\left(2^{-n}\right)^{k}: B\left(x_{0}, r\right) \rightarrow B\left(x_{0}, \alpha\left(r, k 2^{-n}\right)\right) \quad \text { for all } k \in \mathbb{N} \text { and } r \geq 0 .
$$

For $k=1$, the claim holds by Assumption 2.1(ii). For the induction step, suppose that inequality (2.3) holds for some fixed $k \in \mathbb{N}$ and all $r \geq 0$. We combine this with Assumption 2.1(ii) and inequality (2.1) to conclude that

$$
I\left(2^{-n}\right)^{k+1} x=I\left(2^{-n}\right)^{k} I\left(2^{-n}\right) x \in B\left(x_{0}, \alpha\left(\alpha\left(r, 2^{-n}\right), k 2^{-n}\right)\right) \subset B\left(x_{0}, \alpha\left(r,(k+1) 2^{-n}\right)\right.
$$

for all $r \geq 0$ and $x \in B\left(x_{0}, r\right)$.

Second, we show by induction that, for all $k \in \mathbb{N}, r \geq 0$ and $x, y \in B\left(x_{0}, r\right)$,

$$
d\left(I\left(2^{-n}\right)^{k} x, I\left(2^{-n}\right)^{k} y\right) \leq e^{k 2^{-n} \omega_{\alpha\left(r, k 2^{-n}\right)}} d(x, y)
$$


For $k=1$, the claim follows from Assumption 2.1(iii) and $\alpha\left(r, 2^{-n}\right) \geq r$. For the induction step, suppose that inequality (2.4) holds for some fixed $k \in \mathbb{N}$, all $r \geq 0$ and $x, y \in B\left(x_{0}, r\right)$. Combing this with Assumption 2.1(ii) and (iii), inequality (2.1), and the non-decreasingness of the mappings $r \mapsto \omega_{r}$ and $t \mapsto \alpha(r, t)$ yields

$$
\begin{aligned}
& d\left(I\left(2^{-n}\right)^{k+1} x, I\left(2^{-n}\right)^{k+1} y\right)=d\left(I\left(2^{-n}\right)^{k} I\left(2^{n}\right) x, I\left(2^{-n}\right)^{k} I\left(2^{-n}\right) y\right)
\end{aligned}
$$

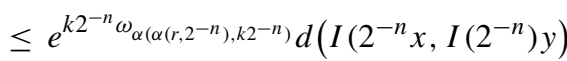

$$
\begin{aligned}
& \leq e^{k 2^{-n} \omega_{\alpha\left(r,(k+1) 2^{-n}\right)}} e^{2^{-n} \omega_{\alpha\left(r, 2^{-n}\right)}} d(x, y) \\
& \leq e^{(k+1) 2^{-n} \omega_{\alpha\left(r,(k+1) 2^{-n}\right)}} d(x, y)
\end{aligned}
$$

for all $r \geq 0$ and $x, y \in B\left(x_{0}, r\right)$.

By definition, for every $s, t \in \mathcal{T}$ and $n \in \mathbb{N}$ with $2^{n} s, 2^{n} t \in \mathbb{N}$,

$$
I\left(\pi_{n}^{s+t}\right)=I\left(\pi_{n}^{s}\right) I\left(\pi_{n}^{t}\right) .
$$

Lemma 2.8 Let $r \geq 0$ and $x \in B\left(x_{0}, r\right) \cap \mathcal{L}^{I}$. Choose $t_{0}>0$ and $c \geq 0$ such that

$$
d(I(t) x, x) \leq c t \quad \text { for all } t \in\left[0, t_{0}\right] .
$$

Then, for every $T \geq 0, s, t \in[0, T] \cap \mathcal{T}$ and $n \in \mathbb{N}$ with $2^{n} s, 2^{n} t \in \mathbb{N}$ and $2^{-n} \leq t_{0}$,

$$
\left.d\left(I\left(\pi_{n}^{s}\right) x, I\left(\pi_{n}^{t}\right) x\right) \leq c e^{T \omega_{\alpha(r, T)}} \mid s-t\right] .
$$

Proof Fix $n \in \mathbb{N}$ with $2^{-n} \leq t_{0}$. First, we show by induction that

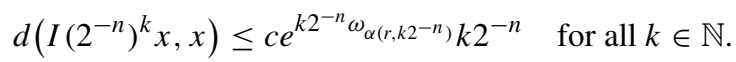

For $k=1$, the claim holds by inequality (2.6). For the induction step, assume that inequality (2.7) holds for some fixed $k \in \mathbb{N}$. We combine this with Lemma 2.7, inequality (2.6), inequality (2.1), and the non-decreasingness of the mappings $r \mapsto \omega_{r}$ and $t \mapsto \alpha(r, t)$ to estimate

$$
\begin{aligned}
& d\left(I\left(2^{-n}\right)^{k+1} x, x\right) \\
& \leq d\left(I\left(2^{-n}\right)^{k} I\left(2^{-n}\right) x, I\left(2^{-n}\right)^{k} x\right)+d\left(I\left(2^{-n}\right)^{k} x, x\right) \\
& \leq e^{k 2^{-n} \omega_{\alpha\left(\alpha\left(r, 2^{-n}\right), k 2^{-n}\right)}} d\left(I\left(2^{-n}\right) x, x\right)+c e^{k 2^{-n} \omega_{\alpha\left(r, k 2^{-n}\right)}} k 2^{-n} \\
& \leq c e^{k 2^{-n} \omega_{\alpha\left(r,(k+1) 2^{-n}\right)}} e^{2^{-n} \omega_{\alpha\left(r, 2^{-n}\right)} 2^{-n}+c e^{k 2^{-n} \omega_{\alpha\left(r, k 2^{-n}\right)}} k 2^{-n}} \\
& \leq c e^{(k+1) 2^{-n} \omega_{\alpha\left(r,(k+1) 2^{-n}\right)}}(k+1) 2^{-n} .
\end{aligned}
$$

Second, we show that, for all $k, l \in \mathbb{N}$ with $k \geq l$,

$$
d\left(I\left(2^{-n}\right)^{k} x, I\left(2^{-n}\right)^{l} x\right) \leq c e^{\left(k 2^{-n} \omega_{\alpha\left(r, k 2^{-n}\right)}(k-l) 2^{-n} .\right.}
$$

It follows from Eq. (2.5), Lemma 2.7, inequality (2.1), and the non-decreasingness of the mappings $r \mapsto \omega_{r}$ and $t \mapsto \alpha(r, t)$ that

$$
\begin{aligned}
d\left(I\left(2^{-n}\right)^{k} x, I\left(2^{-n}\right)^{l} x\right) & =d\left(I\left(2^{-n}\right)^{l} I\left(2^{-n}\right)^{k-l} x, I\left(2^{-n}\right)^{l} x\right) \\
& \leq e^{\left.l 2^{-n} \omega_{\alpha\left(\alpha(r, k-l) 2^{-n}\right), l 2^{-n}}\right)} d\left(I\left(2^{-n}\right)^{k-l} x, x\right) \\
& \leq c e^{l 2^{-n} \omega_{\alpha\left(r, k 2^{-n}\right)}} e^{(k-l) 2^{-n} \omega_{\alpha\left(r,(k-l) 2^{-n}\right)}(k-l) 2^{-n}} \\
& \leq c e^{k 2^{-n} \omega_{\alpha\left(r, k 2^{-n}\right)}}(k-l) 2^{-n}
\end{aligned}
$$


By using Assumption 2.4 and choosing a diagonal sequence for the countable set $\mathcal{D} \times \mathcal{T}$, there exists a subsequence $\left(n_{l}\right)_{l \in \mathbb{N}} \subset \mathbb{N}$ such that the limit

$$
S(t) x:=\lim _{l \rightarrow \infty} I\left(\pi_{n_{l}}^{t}\right) x \in X
$$

exists for all $(x, t) \in \mathcal{D} \times \mathcal{T}$.

Lemma 2.9 1. For every $r \geq 0$ and $x \in B\left(x_{0}, r\right) \cap \mathcal{D}$, there exists $c \geq 0$ such that

$$
\left.d(S(s) x, S(t) x) \leq c e^{T \omega_{\alpha(r, T)}} \mid s-t\right] \quad \text { for all } T \geq 0 \text { and } s, t \in[0, T] \cap \mathcal{T} .
$$

In particular, the mapping $S(\cdot) x:[0, T] \cap \mathcal{T} \rightarrow X$ has a unique continuous extension to $[0, T]$, which satisfies the previous inequality for all $s, t \in[0, T]$.

2. For every $r, t \geq 0$,

$$
d(S(t) x, S(t) y) \leq e^{t \omega_{\alpha(r, t)}} d(x, y) \quad \text { for all } x, y \in B\left(x_{0}, r\right) \cap \mathcal{D} .
$$

In particular, the mapping $S(t): B\left(x_{0}, r\right) \cap \mathcal{D} \rightarrow X$ has a unique continuous extension to $B\left(x_{0}, r\right)$, which satisfies the previous inequality for all $x, y \in B\left(x_{0}, r\right)$.

Proof First, we fix $r, T \geq 0, x \in B\left(x_{0}, r\right) \cap \mathcal{D}$, and choose $t_{0}>0$ and $c \geq 0$ such that

$$
d(I(t) x, x) \leq c t \quad \text { for all } t \in\left[0, t_{0}\right] .
$$

It follows from Eq. (2.8) and Lemma 2.8 that, for all $s, t \in[0, T] \cap \mathcal{T}$,

$$
d(S(s) x, S(t) x)=\lim _{l \rightarrow \infty} d\left(I\left(\pi_{n_{l}}^{s}\right) x, I\left(\pi_{n_{l}}^{t}\right) x\right) \leq c e^{\left.T \omega_{\alpha(r, T)} \mid s-t\right] .}
$$

The existence and uniqueness of the extension follows, because $[0, T] \cap \mathcal{T} \subset[0, T]$ is dense and the mapping $S(\cdot) x:[0, T] \cap \mathcal{T} \rightarrow X$ is Lipschitz continuous.

Second, we fix $r \geq 0$ and $x, y \in B\left(x_{0}, r\right) \cap \mathcal{D}$. It follows from Eq. (2.8) and Lemma 2.7 that

$$
d(S(t) x, S(t) y)=\lim _{l \rightarrow \infty}\left(I\left(\pi_{n_{l}}^{t}\right) x, I\left(\pi_{n_{l}}^{t}\right) y\right) \leq e^{t \omega_{\alpha(r, t)}} d(x, y) \quad \text { for all } t \in \mathcal{T} .
$$

Now, let $t \geq 0$ be arbitrary and choose a sequence $\left(t_{n}\right)_{n \in \mathbb{N}} \subset[0, t] \cap \mathcal{T}$ with $t_{n} \rightarrow t$. We use part (i), inequality (2.9) and the non-decreasingness of $\alpha$ in the second argument to estimate

$$
d(S(t) x, S(t) y)=\lim _{n \rightarrow \infty} d\left(S\left(t_{n}\right) x, S\left(t_{n}\right) y\right) \leq \sup _{n \in \mathbb{N}} e^{t_{n} \omega_{\alpha\left(r, t_{n}\right)}} d(x, y) \leq e^{t \omega_{\alpha(r, t)}} d(x, y) .
$$

The existence and uniqueness of the extension follows, because $B\left(x_{0}, r\right) \cap \mathcal{D} \subset B\left(x_{0}, r\right)$ is dense and the mapping $S(t): B\left(x_{0}, r\right) \cap \mathcal{D} \rightarrow X$ is Lipschitz continuous.

Lemma 2.10 The mapping $S(\cdot) x:[0, \infty) \rightarrow X$ is continuous for all $x \in X$.

Proof Let $x \in X, t \geq 0$ and $\varepsilon>0$. Define $r:=d\left(x_{0}, x\right)+1, T:=t+1$ and choose $\delta_{1} \in(0,1]$ with $2 e^{T \omega_{\alpha(r, T)}} \delta_{1}<\varepsilon / 2$. Since $\mathcal{D} \subset X$ is dense, there exists $y \in B\left(x_{0}, \delta_{1}\right) \cap \mathcal{D}$. Moreover, by Lemma 2.9(i), there exists $c \geq 0$ such that

$$
d\left(S\left(s_{1}\right) y, S\left(s_{2}\right) y\right) \leq e^{T \omega_{\alpha(r, T)}}\left|s_{1}-s_{2}\right| \text { for all } s_{1}, s_{2} \in[0, T] .
$$


Choose $\delta_{2} \in\left(0, \delta_{1}\right]$ with $c e^{T \omega_{\alpha(r, T)}} \delta_{2}<\varepsilon / 2$. For every $s \geq 0$ with $|s-t|<\delta_{2}$, we obtain

$$
\begin{aligned}
d(S(s) x, S(t) x) & \leq d(S(s) x, S(s) y)+d(S(t) x, S(t) y)+d(S(s) y, S(t) y) \\
& \leq 2 e^{T \omega_{\alpha(r, T)}} d(x, y)+c e^{T \omega_{\alpha(r, T)}}|s-t| \\
& \leq 2 e^{T \omega_{\alpha(r, T)}} \delta_{1}+c e^{T \omega_{\alpha(r, T)}} \delta_{2}<\varepsilon .
\end{aligned}
$$

Lemma 2.11 It holds $S(t) x=\lim _{l \rightarrow \infty} I\left(\pi_{n_{l}}^{t}\right) x$ for all $x \in X$ and $t \in \mathcal{T}$. In particular, we obtain $S(t): B\left(x_{0}, r\right) \rightarrow B\left(x_{0}, \alpha(r, t)\right)$ for all $r, t \geq 0$.

Proof First, let $x \in X, t \in \mathcal{T}, \varepsilon>0$ and define $r:=d\left(x_{0}, x\right)+1$. Choose $\delta>0$ with $2 e^{t \omega_{\alpha(r, t)}} \delta<\varepsilon$ and $y \in B(x, \delta) \cap \mathcal{D}$. For every $l \in \mathbb{N}$ with $2^{n_{l}} t \in \mathbb{N}$, we use Lemmas 2.7 and 2.9(ii) to estimate

$$
\begin{aligned}
d\left(S(t) x, I\left(\pi_{n_{l}}^{t}\right) x\right) & \leq d(S(t) x, S(t) y)+d\left(I\left(\pi_{n_{l}}^{t}\right) x, I\left(\pi_{n_{l}}^{t}\right) y\right)+d\left(S(t) y, I\left(\pi_{n_{l}}^{t}\right) y\right) \\
& \leq 2 e^{t \omega_{\alpha(r, t)}} d(x, y)+d\left(S(t) y, I\left(\pi_{n_{l}}^{t}\right) y\right) \\
& <\varepsilon+d\left(S(t) y, I\left(\pi_{n_{l}}^{t}\right) y\right) .
\end{aligned}
$$

Equation (2.8) implies $\lim _{l \rightarrow \infty} d\left(S(t) x, I\left(\pi_{n_{l}}^{t}\right) x\right)=0$.

Second, we fix $r \geq 0$ and $x \in B\left(x_{0}, r\right)$. It follows from the first part and Lemma 2.7 that

$$
d\left(x_{0}, S(t) x\right)=\lim _{l \rightarrow \infty} d\left(x_{0}, I\left(\pi_{n_{l}}^{t}\right) x\right) \leq \alpha(r, t) \quad \text { for all } t \in \mathcal{T} .
$$

Now, let $t \geq 0$ be arbitrary and choose a sequence $\left(t_{n}\right)_{n \in \mathbb{N}} \subset[0, t] \cap \mathcal{T}$ with $t_{n} \rightarrow t$. We use Lemma 2.10, inequality (2.10) and the non-decreasingness of $\alpha$ in the second argument to conclude that

$$
d\left(x_{0}, S(t) x\right)=\lim _{n \rightarrow \infty} d\left(x_{0}, S\left(t_{n}\right) x\right) \leq \sup _{n \in \mathbb{N}} \alpha\left(r, t_{n}\right) \leq \alpha(r, t) .
$$

Lemma 2.12 It holds $S(0)=\mathrm{id}_{X}$ and $S(s+t)=S(s) S(t)$ for all $s, t \geq 0$.

Proof It follows from Assumption 2.1(i) and the construction that $S(0)=\operatorname{id}_{X}$. Fix $x \in X$ and define $r:=d\left(x_{0}, x\right)$. First, let $s, t \in \mathcal{T}$. Equation (2.5) and Lemma 2.11 imply

$$
\lim _{l \rightarrow \infty} d\left(S(s+t) x, I\left(\pi_{n_{l}}^{s}\right) I\left(\pi_{n_{l}}^{t}\right) x\right)=\lim _{l \rightarrow \infty} d\left(S(s+t) x, I\left(\pi_{n_{l}}^{s+t}\right) x\right)=0 .
$$

Furthermore, it follows from Lemmas 2.7 and 2.11 that

$$
\begin{aligned}
& d\left(S(s) S(t) x, I\left(\pi_{n_{l}}^{s}\right) I\left(\pi_{n_{l}}^{t}\right) x\right) \\
& \leq d\left(S(s) S(t) x, I\left(\pi_{n_{l}}^{s}\right) S(t) x\right)+d\left(I\left(\pi_{n_{l}}^{s}\right) S(t) x, I\left(\pi_{n_{l}}^{s}\right) I\left(\pi_{n_{l}}^{t}\right) x\right) \\
& \leq d\left(S(s) S(t) x, I\left(\pi_{n_{l}}^{s}\right) S(t) x\right)+e^{s \omega_{\alpha(\alpha(r, t), s)}} d\left(S(t) x, I\left(\pi_{n_{l}}^{t}\right) \rightarrow 0 \text { as } l \rightarrow \infty .\right.
\end{aligned}
$$


Second, let $s, t \geq 0$ be arbitrary, define $T:=s+t+1$ and choose sequences $\left(s_{n}\right)_{n \in \mathbb{N}}$ and $\left(t_{n}\right)_{n \in \mathbb{N}}$ in $[0, T] \cap \mathcal{T}$ with $s_{n} \rightarrow s$ and $t_{n} \rightarrow t$. We use the first part, Lemma 2.9(ii), Lemmas 2.10 and 2.11 to estimate

$$
\begin{aligned}
& d(S(s+t) x, S(s) S(t) x) \\
& \leq d\left(S(s+t) x, S\left(s_{n}+t_{n}\right) x\right)+d\left(S(s) S(t) x, S\left(s_{n}\right) S(t) x\right) \\
& \quad+d\left(S\left(s_{n}\right) S(t) x, S\left(s_{n}\right) S\left(t_{n}\right) x\right) \\
& \leq d\left(S(s+t) x, S\left(s_{n}+t_{n}\right) x\right)+d\left(S(s) S(t) x, S\left(s_{n}\right) S(t) x\right) \\
& \quad+e^{T \omega_{\alpha(\alpha(r, T), T)}} d\left(S(t) x, S\left(t_{n}\right) x\right) \rightarrow 0 \quad \text { as } n \rightarrow \infty .
\end{aligned}
$$

Lemma 2.13 For every $r \geq 0$ and $x \in B\left(x_{0}, r\right) \cap \mathcal{L}^{I}$, there exists $c \geq 0$ such that

$$
d(S(s) x, S(t) x) \leq c e^{T \omega_{\alpha(r, T)}}|s-t| \text { for all } T \geq 0 \text { and } s, t \in[0, T] .
$$

Proof Fix $r, T \geq 0, x \in B\left(x_{0}, r\right) \cap \mathcal{L}^{I}$ and choose $t_{0}>0$ and $c \geq 0$ such that

$$
d(I(t) x, x) \leq c t \quad \text { for all } t \in\left[0, t_{0}\right] .
$$

It follows from Lemmas 2.11 and 2.8 that, for all $s, t \in[0, T] \cap \mathcal{T}$,

$$
d(S(s) x, S(t) x)=\lim _{l \rightarrow \infty} d\left(I\left(\pi_{n_{l}}^{s}\right) x, I\left(\pi_{n_{l}}^{t}\right) x\right) \leq c e^{T \omega_{\alpha(r, T)}}|s-t| .
$$

Now, let $s, t \in[0, T]$ be arbitrary and choose sequences $\left(s_{n}\right)_{n \in \mathbb{N}}$ and $\left(t_{n}\right)_{n \in \mathbb{N}}$ in $[0, T] \cap \mathcal{T}$ with $s_{n} \rightarrow s$ and $t_{n} \rightarrow t$. We use Lemma 2.10 and inequality (2.11) to conclude that

$$
d(S(s) x, S(t) x)=\lim _{n \rightarrow \infty} d\left(S\left(s_{n}\right) x, S\left(t_{n}\right) x\right) \leq c e^{T \omega_{\alpha(r, T)}}|s-t| .
$$

\subsection{Discussion and Comparison with Nisio Semigroup}

In the previous subsection, we only considered dyadic partitions, but actually the same iterations can be made with arbitrary partitions. The proofs do not change, except for being notationally more complicated. For every $t \geq 0$, we denote by $P_{t}$ the set of all partitions $\pi=\left\{t_{0}, \ldots, t_{n}\right\}$ with $0=t_{0}<t_{1}<\ldots<t_{n}=t$. Moreover, we define the iterated operators

$$
I(\pi):=I\left(t_{1}-t_{0}\right) \cdots I\left(t_{n}-t_{n-1}\right) .
$$

For later reference, we state the following version of Lemmas 2.7 and 2.8.

Remark 2.14 Let $(I(t))_{t \geq 0}$ be a family of operators, which satisfy Assumption 2.1. Then, the following statements hold:

1. For every $r, t \geq 0$ and $\pi \in P_{t}$,

$$
\begin{aligned}
d\left(x_{0}, I(\pi) x\right) & \leq \alpha(r, t) \quad \text { for all } x \in B\left(x_{0}, r\right), \\
d(I(\pi) x, I(\pi) y) & \leq e^{t \omega_{\alpha(r, t)}} d(x, y) \quad \text { for all } x, y \in B\left(x_{0}, r\right) .
\end{aligned}
$$

2. Let $r \geq 0$ and $x \in B(0, r) \cap \mathcal{L}^{I}$. Choose $t_{0}>0$ and $c \geq 0$ with

$$
d(I(t) x, x) \leq c t \quad \text { for all } t \in\left[0, t_{0}\right] .
$$


Then, for all $t \geq 0$ and $\pi \in P_{t}$ with $|\pi| \leq t_{0}$,

$$
d(I(\pi) x, x) \leq c e^{t \omega_{\alpha(r, t)} t},
$$

where $|\pi|:=\max _{i=0, \ldots, n-1}\left(s_{i+1}-s_{i}\right)$ for $\pi=\left\{s_{0}, \ldots, s_{n}\right\}$.

A priori the construction of an associated semigroup $(S(t))_{t \geq 0}$ to a generating family $(I(t))_{t \geq 0}$ depends on the choice of the partitions and the convergent subsequence. However, it is possible that

$$
S(t) x=\lim _{n \rightarrow \infty} I\left(\pi_{n}^{t}\right) x \quad \text { for all }(x, t) \in X \times \mathcal{T},
$$

i.e., the convergence holds without choosing a subsequence. For instance, if the sequence $\left(I\left(\pi_{n}^{t}\right) x\right)_{n \in \mathbb{N}}$ is non-decreasing for all $(x, t) \in X \times \mathcal{T}$, we obtain

$$
S(t) x=\sup _{l \in \mathbb{N}} I\left(\pi_{n_{l}}^{t}\right) x=\sup _{n \in \mathbb{N}} I\left(\pi_{n}^{t}\right) x \quad \text { for all }(x, t) \in X \times \mathcal{T} .
$$

In particular, Nisio semigroups fall into this category, as we will see in the remainder of this subsection. Furthermore, if the semigroup represents the unique solution to a PDE, the construction is independent of the choice of the convergent subsequence. For details, we refer to Section 4.2. For the following lemma and subsequent remark, let $X$ be a Banach lattice. An operator $I(t): X \rightarrow X$ is called

- monotone, if $I(t) x \leq I(t) y$ for all $x, y \in X$ with $x \leq y$,

- continuous from below, if $I(t) x=\sup _{n \in \mathbb{N}} I(t) x_{n}$ for every non-decreasing sequence $\left(x_{n}\right)_{n \in \mathbb{N}} \subset X$ such that $x:=\sup _{n \in \mathbb{N}} x_{n} \in X$ exists.

Lemma 2.15 Let $(I(t))_{t \geq 0}$ be a family of operators $I(t): X \rightarrow X$ satisfying Assumptions 2.1 and 2.4, and $(S(t))_{t \geq 0}$ an associated semigroup as in Theorem 2.5. In addition, we make the following assumptions:

(i) $I(t)$ is monotone and continuous from below for all $t \geq 0$.

(ii) $I(s+t) x \leq I(s) I(t) x$ for all $s, t \geq 0$ and $x \in X$.

(iii) The mapping $\mathbb{R}_{+} \rightarrow X, t \mapsto I(t) x$ is continuous for all $x \in X$.

(iv) The operator $T(t): X \rightarrow X, x \mapsto \sup _{\pi \in P_{t}} I(\pi) x$ is well-defined for all $t \geq 0$.

Then, it holds $S(t) x=T(t) x$ for all $(x, t) \in X \times \mathbb{R}_{+}$. Furthermore,

$$
S(t) x=\lim _{n \rightarrow \infty} I\left(\pi_{n}^{t}\right) x \quad \text { for all }(x, t) \in X \times \mathcal{T},
$$

i.e., the convergence holds without choosing a subsequence.

Proof By induction, it follows from condition (ii) that the sequence $\left(I\left(\pi_{n}^{t}\right) x\right)_{n \in \mathbb{N}}$ is nondecreasing for all $(x, t) \in X \times \mathcal{T}$. Moreover, by Theorem 2.5, there exists a subsequence $\left(n_{l}\right)_{l \in \mathbb{N}} \subset \mathbb{N}$ such that $S(t) x=\lim _{l \rightarrow \infty} I\left(\pi_{n_{l}}^{t}\right) x$ for all $(x, t) \in X \times \mathcal{T}$. Since $X$ is a Banach lattice, we obtain

$$
I(t) x \leq S(t) x=\sup _{l \in \mathbb{N}} I\left(\pi_{n_{l}}^{t}\right) x=\sup _{n \in \mathbb{N}} I\left(\pi_{n}^{t}\right) x \leq T(t) x \quad \text { for all }(x, t) \in X \times \mathcal{T} .
$$

In addition, condition (iii) and strong continuity of $(S(t))_{t \geq 0}$ imply $I(t) x \leq S(t) x$ for all $(x, t) \in X \times \mathbb{R}_{+}$. We use the monotonicity of $I(s)$ and the semigroup property of $(S(t))_{t \geq 0}$ to conclude

$$
I(s) I(t) x \leq I(s) S(t) x \leq S(s) S(t) x=S(s+t) x \quad \text { for all } s, t \geq 0 \text { and } x \in X .
$$


By induction, we obtain $T(t) x \leq S(t) x$ for all $(x, t) \in X \times \mathbb{R}_{+}$with equality for $t \in \mathcal{T}$. It remains to show that the mapping $\mathbb{R}_{+} \rightarrow X, t \mapsto T(t) x$ is continuous for all $x \in X$. Condition (ii) implies that the set $\left\{I(\pi): \pi \in P_{t}\right\}$ is directed upwards and, by assumption, the operator $I(t)$ is continuous from below for all $t \geq 0$. Hence, the family $(T(t))_{t \geq 0}$ forms a semigroup, and we can use Remark 2.14(ii) to show that the mapping $\mathbb{R}_{+} \rightarrow X, t \mapsto T(t) x$ is locally Lipschitz continuous for all $x \in \mathcal{L}^{I}$. Since $\mathcal{L}^{I} \subset X$ is dense, it follows from Remark 2.14(i) that the mapping $t \mapsto T(t) x$ is continuous for all $x \in X$, see the proof of Lemma 2.10. Furthermore, it holds $S(t) x=\lim _{n \rightarrow \infty} I\left(\pi_{n}^{t}\right) x$ for all $(x, t) \in X \times \mathcal{T}$, because the limit along a subsequence in Theorem 2.5(i) does not depend on the choice of the convergent subsequence.

Remark 2.16 Let $\left(S_{\lambda}\right)_{\lambda \in \Lambda}$ be a family of linear semigroups on $X$, which satisfy the following conditions:

(i) $S_{\lambda}(t)$ is monotone and continuous from below for all $\lambda \in \Lambda$ and $t \geq 0$.

(ii) There exists $\omega \geq 0$ such that $\left\|S_{\lambda}(t) x\right\| \leq e^{\omega t}\|x\|$ for all $\lambda \in \Lambda, t \geq 0$ and $x \in X$.

(iii) The operator $I(t): X \rightarrow X, x \mapsto \sup _{\lambda \in \Lambda} S_{\lambda}(t) x$ is well-defined for all $t \geq 0$.

Moreover, we assume that, for every subset $Y \subset X$ such that the supremum $\sup Y \in X$ exists, it holds $\|\sup Y\| \leq \sup _{x \in Y}\|x\|$. For instance, the supremum norm has this property, but not the $L^{p}$-norm. For every $t \geq 0$ and $x, y \in X$, we use the assumption on the norm and condition (ii) to estimate

$$
\begin{aligned}
\|I(t) x\| & \leq \sup _{\lambda \in \Lambda}\left\|S_{\lambda}(t) x\right\| \leq e^{\omega t}\|x\|, \\
\|I(t) x-I(t) y\| & \leq \sup _{\lambda \in \Lambda}\left\|S_{\lambda}(t) x-S_{\lambda}(t) y\right\| \leq e^{\omega t}\|x-y\| .
\end{aligned}
$$

Hence, Assumption 2.1 is satisfied. Furthermore, condition (i) implies that $(I(t))_{t \geq 0}$ satisfies the first two conditions of Lemma 15. In many examples, the forth condition of Lemma 15 follows from the assumptions, which are already necessary for the construction of the semigroup $(S(t))_{t \geq 0}$, while the third condition requires a mild additional assumption. If $(I(t))_{t \geq 0}$ satisfies the assumptions of Lemma 15, the associated semigroup $(S(t))_{t \geq 0}$ from Theorem 2.5 equals the family $(T(t))_{t \geq 0}$, defined by

$$
T(t) x:=\sup _{\pi \in P_{t}} I(\pi) x \quad \text { for all }(x, t) \in X \times \mathbb{R}_{+} .
$$

It is also possible to weight the linear semigroups in the definition of $I(t)$ with a penalization term. This leads to semigroups, which are not sublinear but merely convex.

\section{Relative Compactness Based on Arzéla-Ascoli's Theorem}

Let $C$ be the space of all continuous functions $f: \mathbb{R}^{d} \rightarrow \mathbb{R}^{m}$, including the subsets $C^{\infty}$, Lip and $C_{0}$ of all functions, which are infinitely differentiable, Lipschitz continuous and vanish at infinity, respectively. Furthermore, let $\mathcal{L}^{\infty}$ be the space of all bounded (not necessarily measurable) functions $f: \mathbb{R}^{d} \rightarrow \mathbb{R}^{m}$, endowed with the supremum norm $\|f\|_{\infty}:=\sup _{x \in \mathbb{R}^{d}}|f(x)|$, where $|\cdot|$ denotes the Euclidean norm. We define $C_{b}:=C \cap \mathcal{L}^{\infty}$, $\operatorname{Lip}_{b}:=\operatorname{Lip} \cap \mathcal{L}^{\infty}$ and $\operatorname{Lip}_{0}:=\operatorname{Lip} \cap C_{0}$. In addition, for every $c \geq 0$, we denote by $\operatorname{Lip}(c)$ the set of all $c$-Lipschitz continuous functions. For every $c \geq 0$, let

$$
\operatorname{Lip}_{b}(c):=\left\{f \in \operatorname{Lip}_{b}: f \in \operatorname{Lip}(c),\|f\|_{\infty} \leq c\right\} \quad \text { and } \quad \operatorname{Lip}_{0}(c)=\operatorname{Lip}_{b}(c) \cap C_{0} .
$$




\subsection{Semigroups on $C_{0}$}

We give explicit conditions, how to verify the assumptions of Section 2 for a family of translation-invariant contractions, which will be illustrated in Section 6. We start with an application of Arzéla-Ascoli's theorem.

Lemma 3.1 Let $(I(t))_{t \geq 0}$ be a family of operators $I(t): C_{0} \rightarrow C_{0}$, which satisfy Assumption 2.1(ii). Let $t \in \mathcal{T}$ and $f \in C_{0}$ such that

- $\left\{I\left(\pi_{n}^{t}\right) f: n \in \mathbb{N}\right\}$ is equicontinuous,

- $\lim _{|x| \rightarrow \infty} \sup _{n \in \mathbb{N}}\left|\left(I\left(\pi_{n}^{t}\right) f\right)(x)\right|=0$.

Then, the sequence $\left(I\left(\pi_{n}^{t}\right) f\right)_{n \in \mathbb{N}}$ is relatively compact in $C_{0}$.

Proof By assumption, the sequence $\left(I\left(\pi_{n}^{t}\right)_{n \in \mathbb{N}}\right.$ is equicontinuous. Moreover, it follows from Lemma 2.7 that $\left(I\left(\pi_{n}^{t}\right)_{n \in \mathbb{N}}\right.$ is bounded. Note that Lemma 2.7 is a consequence of Assumption 2.1(ii) and independent of the other assumptions in Section 2. By using ArzélaAscoli's theorem and choosing a diagonal sequence, we obtain a function $g \in C$ such that $I\left(\pi_{n_{l}}^{t}\right) f \rightarrow g$ as $l \rightarrow \infty$ uniformly on compact sets for a suitable subsequence. It remains to show

$$
\lim _{l \rightarrow \infty}\left\|I\left(\pi_{n_{l}}^{t}\right) f-g\right\|_{\infty}=0 .
$$

For every $\varepsilon>0$, by assumption, there exists a compact set $K \subset \mathbb{R}^{d}$ with

$$
\sup _{x \in K^{c}} \sup _{n \in \mathbb{N}}\left|\left(I\left(\pi_{n}^{t}\right) f\right)(x)\right| \leq \frac{\varepsilon}{2} .
$$

This inequality is preserved in the limit, i.e., $\sup _{x \in K^{c}}|g(x)| \leq \varepsilon / 2$. We obtain

$$
\begin{aligned}
\left\|I\left(\pi_{n_{l}}^{t}\right) f-g\right\|_{\infty} & =\left\|\left(I\left(\pi_{n_{l}}^{t}\right) f-g\right) \mathbb{1}_{K}\right\|_{\infty}+\left\|\left(I\left(\pi_{n_{l}}^{t}\right) f-g\right) \mathbb{1}_{K^{c}}\right\|_{\infty} \\
& \leq\left\|\left(I\left(\pi_{n_{l}}^{t}\right) f-g\right) \mathbb{1}_{K}\right\|_{\infty}+\varepsilon \rightarrow \varepsilon \quad \text { as } l \rightarrow \infty .
\end{aligned}
$$

In addition, since $C_{0}$ is complete, it holds $g \in C_{0}$.

An operator $I(t): C_{0} \rightarrow C_{0}$ is called translation-invariant, if

$$
(I(t) f)(x)=\left(I(t) f_{x}\right)(0) \text { for all } f \in C_{0} \text { and } x \in \mathbb{R}^{d},
$$

where $f_{x}: \mathbb{R}^{d} \rightarrow \mathbb{R}^{m}, y \mapsto f(x+y)$. Let $C_{0}^{+}:=\left\{f \in C_{0}: f \geq 0\right\}$.

Lemma 3.2 Let $(I(t))_{t \geq 0}$ be a family of operators $I(t): C_{0} \rightarrow \mathcal{L}^{\infty}$, which satisfy the following conditions:

(i) $I(0)=\operatorname{id}_{C_{0}}$.

(ii) $\|I(t) f\|_{\infty} \leq\|f\|_{\infty}$ for all $t \geq 0$ and $f \in C_{0}$.

(iii) $\|I(t) f-I(t) g\|_{\infty} \leq\|f-g\|_{\infty}$ for all $t \geq 0$ and $f, g \in C_{0}$.

(iv) $I(t)$ is translation-invariant for all $t \geq 0$.

(v) There exists a countable set $\mathcal{D} \subset \operatorname{Lip}_{0} \cap \mathcal{L}^{I}$, which is dense in $C_{0}$.

(vi) For every $c \geq 0$, there exists a family $\left(T_{c}(t)\right)_{t \geq 0}$ of operators $T_{c}(t): C_{0}^{+} \rightarrow C_{0}^{+}$such that

- $|I(t) f| \leq T_{c}(t)|f|$ for all $f \in \operatorname{Lip}_{0}(c)$ and $t \geq 0$,

- $T_{c}(s) T_{c}(t) f \leq T_{c}(s+t) f$ for all $f \in C_{0}^{+}$and $s, t \geq 0$,

- $T_{c}(t)$ is monotone for all $t \geq 0$. 
Then, it holds $I(t): C_{0} \rightarrow C_{0}$ and $I(t): \operatorname{Lip}_{0}(c) \rightarrow \operatorname{Lip}_{0}(c)$ for all $c, t \geq 0$. Furthermore, the family $(I(t))_{t \geq 0}$ satisfies Assumptions 2.1 and 2.4 .

Proof First, we show that $I(t): \operatorname{Lip}_{0}(c) \rightarrow \operatorname{Lip}_{0}(c)$ for all $c, t \geq 0$. Fix $c, t \geq 0$ and $f \in \operatorname{Lip}_{0}(c)$. For every $x, y \in \mathbb{R}^{d}$, condition (iii) and (iv) imply

$$
|(I(t) f)(x)-(I(t) f)(y)|=\left|\left(I(t) f_{x}-I(t) f_{y}\right)(0)\right| \leq\left\|f_{x}-f_{y}\right\|_{\infty} \leq c|x-y|,
$$

and therefore $I(t) f \in \operatorname{Lip}_{b}(c)$ by condition (ii). Moreover, condition (vi) yields

$$
\lim _{|x| \rightarrow \infty}|(I(t) f)(x)| \leq \lim _{|x| \rightarrow \infty}\left(T_{c}(t)|f|\right)(x)=0 .
$$

We obtain $I(t) f \in \operatorname{Lip}_{0}(c)$ and conclude $I(t): C_{0} \rightarrow C_{0}$, since $\overline{\operatorname{Lip}_{0}}=C_{0}$, the mapping $I(t): C_{0} \rightarrow \mathcal{L}^{\infty}$ is Lipschitz continuous, and $C_{0} \subset \mathcal{L}^{\infty}$ is closed.

Second, Assumption 2.1 is satisfied, because of condition (i)-(iii). Furthermore, by condition (v), there exists a countable set $\mathcal{D} \subset \operatorname{Lip}_{0} \cap \mathcal{L}^{I}$, which is dense in $C_{0}$. It remains to verify the assumptions from Lemma 3.1 for all $(f, t) \in \mathcal{D} \times \mathcal{T}$. Fix $(f, t) \in \mathcal{D} \times \mathcal{T}$ and choose $c \geq 0$ with $f \in \operatorname{Lip}_{0}(c)$. By induction, it follows from $I\left(2^{-n}\right): \operatorname{Lip}_{0}(c) \rightarrow \operatorname{Lip}_{0}(c)$ that $\left(I\left(\pi_{n}^{t}\right) f\right)_{n \in \mathbb{N}} \subset \operatorname{Lip}_{0}(c)$ for all $n \in \mathbb{N}$ with $2^{n} t \in \mathbb{N}$. In particular, the sequence $\left(I\left(\pi_{n}^{t}\right) f\right)_{n \in \mathbb{N}}$ is equicontinuous. Moreover, we use condition (vi) and $I\left(2^{-n}\right) f \in \operatorname{Lip}_{0}(c)$ to estimate

$$
\left|I\left(2^{-n}\right)^{2} f\right| \leq T_{c}\left(2^{-n}\right)\left(\left|I\left(2^{-n}\right) f\right|\right) \leq T_{c}\left(2^{-n}\right) T_{c}\left(2^{-n}\right)|f| \leq T_{c}\left(2 \cdot 2^{-n}\right)|f| .
$$

By induction, it follows that $\left|I\left(\pi_{n}^{t}\right) f\right| \leq T_{c}(t)|f|$ for all $n \in \mathbb{N}$ with $2^{n} t \in \mathbb{N}$. Hence, condition (vi) implies

$$
\lim _{|x| \rightarrow \infty} \sup _{n \in \mathbb{N}}\left|\left(I\left(\pi_{n}^{t}\right) f\right)(x)\right| \leq \lim _{|x| \rightarrow \infty}\left(T_{c}(t)|f|\right)(x)=0 .
$$

Lemma 3.1 yields that Assumption 2.4 is satisfied.

\subsection{Closure of Lipschitz Functions and Weighted Norms}

To study examples, which are not translation-invariant, the space $\left(C_{0},\|\cdot\|_{\infty}\right)$ is not a suitable choice. Thus, following the setting of Nendel and Röckner [24], we modify the supremum norm with a weight function $\kappa$. The verification of Assumption 2.4 becomes particularly simple, also in the translation-invariant case.

Let $\kappa: \mathbb{R}^{d} \rightarrow(0, \infty)$ be a continuous function function, vanishing at infinity. Let $C_{\kappa}$ be the space of all continuous functions $f: \mathbb{R}^{d} \rightarrow \mathbb{R}^{m}$ such that $\|f \kappa\|_{\infty}<\infty$, endowed with the norm $\|f\|_{\kappa}:=\|f \kappa\|_{\infty}$. Since the mapping

$$
C_{\kappa} \rightarrow C_{b}, f \mapsto f \kappa
$$

is an isomorphism, which is linear, isometric and preserves the pointwise order, the space $C_{\kappa}$ is a Banach lattice. Furthermore, we define $\mathrm{UC}_{\kappa}$ as the closure of $\operatorname{Lip}_{b}$ in $C_{\kappa}$. In particular, the space $\mathrm{UC}_{\kappa}$ is a Banach lattice.

Lemma 3.3 Let $(I(t))_{t \geq 0}$ be a family of operators $I(t): \mathrm{UC}_{\kappa} \rightarrow \mathrm{UC}_{\kappa}$. Assume that there exists a function $\rho: \mathbb{R}_{+} \times \mathbb{R}_{+} \rightarrow \mathbb{R}_{+}$such that

- $\quad I(t): \operatorname{Lip}_{b}(c) \rightarrow \operatorname{Lip}_{b}(\rho(c, t))$ for all $c, t \geq 0$,

- $\rho(\rho(c, s), t) \leq \rho(c, s+t)$ for all $c, s, t \geq 0$.

Then, the sequence $\left(I\left(\pi_{n}^{t}\right) f\right)_{n \in \mathbb{N}}$ is relatively compact in $\mathrm{UC}_{\kappa}$ for all $f \in \operatorname{Lip}_{b}$ and $t \in \mathcal{T}$. 
Proof First, we show that $\operatorname{Lip}_{b}(c) \subset \mathrm{UC}_{\kappa}$ is compact for all $c \geq 0$. Let $c \geq 0$ and $\left(f_{n}\right)_{n \in \mathbb{N}} \subset \operatorname{Lip}_{b}(c)$ be a sequence. By using Arzéla-Ascoli's theorem and choosing a diagonal sequence, we obtain a function $f \in C$ such that $f_{n_{l}} \rightarrow f$ as $l \rightarrow \infty$ uniformly on compact sets for a suitable subsequence. Since $\operatorname{Lip}_{b}(c)$ is closed under mere pointwise convergence, it holds $f \in \operatorname{Lip}_{b}(c)$. It remains to show $\left\|f_{n_{l}}-f\right\|_{\kappa} \rightarrow 0$. Let $\varepsilon>0$ and choose a compact set $K \subset \mathbb{R}^{d}$ with $\sup _{x \in K^{c}} \kappa(x)<\varepsilon / 2 c$. We obtain

$$
\begin{aligned}
\left\|f_{n_{l}}-f\right\|_{\kappa} & =\left\|\left(f_{n_{l}}-f\right) \mathbb{1}_{K}\right\|_{\kappa}+\left\|\left(f_{n_{l}}-f\right) \mathbb{1}_{K^{c}}\right\|_{\infty} \\
& \leq\left\|\left(f_{n_{l}}-f\right) \mathbb{1}_{K}\right\|_{\kappa}+\varepsilon \rightarrow \varepsilon \text { as } l \rightarrow \infty .
\end{aligned}
$$

Second, let $f \in \operatorname{Lip}_{b}(c)$ for some $c \geq 0$ and $t \in \mathcal{T}$. By induction, it follows from the assumptions on $I$ and $\rho$ that $I\left(\pi_{n}^{t}\right) f \in \operatorname{Lip}_{b}(\rho(c, t))$ for all $n \in \mathbb{N}$ with $2^{n} t \in \mathbb{N}$. The first part yields that the sequence $\left(I\left(\pi_{n}^{t}\right) f\right)_{n \in \mathbb{N}}$ is relatively compact in $\mathrm{UC}_{\kappa}$.

Let $C_{c}^{\infty}$ be the space of all infinitely differentiable functions $f: \mathbb{R}^{d} \rightarrow \mathbb{R}^{m}$ with compact support.

Lemma 3.4 Assume that $\phi$ is infinitely differentiable. Then, the space $C_{c}^{\infty} \subset \mathrm{UC}_{\kappa}$ is dense and the mapping $\phi: \mathrm{UC}_{\kappa} \rightarrow C_{0}, f \mapsto f \kappa$ is an isomorphism.

Proof It follows from $\lim _{|x| \rightarrow \infty} \kappa(x)=0$ and the continuity of $\kappa$ that $\phi\left(\operatorname{Lip}_{b}\right) \subset C_{0}$. We conclude $\phi\left(\mathrm{UC}_{\kappa}\right) \subset C_{0}$, because $\phi: \mathrm{UC}_{\kappa} \rightarrow C_{b}$ is isometric and $\operatorname{Lip}_{b} \subset \mathrm{UC}_{\kappa}$ is dense. It remains to show $\phi\left(\mathrm{UC}_{\kappa}\right)=C_{0}$. Let $f \in C_{0}$ and choose a sequence $\left(f_{n}\right)_{n \in \mathbb{N}} \subset C_{c}^{\infty}$ with $\left\|f-f_{n}\right\|_{\infty} \rightarrow 0$. Since $\kappa$ is smooth, it holds $f_{n} / \kappa \in C_{c}^{\infty}$ for all $n \in \mathbb{N}$. Furthermore, the sequence $\left(f_{n} / \kappa\right)_{n \in \mathbb{N}} \subset \mathrm{UC}_{\kappa}$ is a Cauchy sequence and the limit $g:=\lim _{n \rightarrow \infty} f_{n} / \kappa \in \mathrm{UC}_{\kappa}$ exists, because $\phi$ is isometric. We obtain

$$
\phi(g)=\lim _{n \rightarrow \infty} \phi\left(\frac{f_{n}}{\kappa}\right)=\lim _{n \rightarrow \infty} f_{n}=f .
$$

In addition, since $C_{c}^{\infty} \subset C_{0}$ is dense and $\phi$ is isometric, the set $\phi^{-1}\left(C_{c}^{\infty}\right) \subset C_{c}^{\infty} \subset \mathrm{UC}_{\kappa}$ is also dense.

\section{Infinitesimal Generator}

Throughout this section, we assume that $X$ is a Banach space with norm $\|\cdot\|$. We investigate the relation between the local behaviour of a generating family $(I(t))_{t \geq 0}$ and an associated semigroup $(S(t))_{t \geq 0}$. The technical condition (4.2) in Theorem 4.3 will be discussed in Section 4.1.

Assumption 4.1 Let $(I(t))_{t \geq 0}$ be a family of operators, which satisfy Assumption 2.1 with $x_{0}:=0$, and $(S(t))_{t \geq 0}$ a strongly continuous semigroup on $X$. In addition, we assume that

$$
\left\|\frac{S(t) x-x}{t}-y\right\| \leq \sup _{n \in \mathbb{N}}\left\|\frac{I\left(\pi_{n}^{t}\right) x-x}{t}-y\right\| \quad \text { for all } t \in \mathcal{T} \backslash\{0\} \text { and } x, y \in X .
$$

Remark 4.2 If $(I(t))_{t \geq 0}$ satisfies both Assumptions 2.1 and 2.4, and $(S(t))_{t \geq 0}$ is an associated semigroup as in Theorem 2.5, inequality (4.1) is clearly satisfied. But requiring norm convergence $I\left(\pi_{n}^{t}\right) x \rightarrow S(t) x$ is an unnecessarily strong assumption for the next theorem. For instance, if $X$ is a space of continuous functions endowed with the supremum or $\kappa$-norm, 
inequality (4.1) is satisfied if we have only pointwise convergence $I\left(\pi_{n_{l}}^{t}\right) f \rightarrow S(t) f$. In particular, Theorem 4.3 is applicable for Nisio semigroups.

Theorem 4.3 Suppose that Assumption 4.1 holds. Let $x, y \in X$ such that, for every $\varepsilon>0$, there exists $t_{0}>0$ with

$$
\left\|\frac{I\left(2^{-n}\right)^{k}\left(x+2^{-n} y\right)-I\left(2^{-n}\right)^{k} x}{2^{-n}}-y\right\| \leq \varepsilon \quad \text { for all } k, n \in \mathbb{N} \text { with } k 2^{-n} \leq t_{0} .
$$

Then,

$$
\lim _{t \downarrow 0}\left\|\frac{I(t) x-x}{t}-y\right\|=0 \quad \text { implies } \quad \lim _{t \downarrow 0}\left\|\frac{S(t) x-x}{t}-y\right\|=0 .
$$

Proof Fix $\varepsilon>0$ and choose $r \geq 0$ with $x, y \in B(0, r)$. By assumption, there exists $t_{0} \in(0,1]$ such that

$$
\left\|\frac{I(t) x-x}{t}-y\right\| \leq \frac{\varepsilon}{2} e^{-\omega_{\alpha(2 r, 1)}} \quad \text { for all } t \in\left(0, t_{0}\right],
$$

and

$$
\left\|\frac{I\left(2^{-n}\right)^{k}\left(x+2^{-n} y\right)-I\left(2^{-n}\right)^{k} x}{2^{-n}}-y\right\| \leq \frac{\varepsilon}{2} \quad \text { for all } k, n \in \mathbb{N} \text { with } k 2^{-n} \leq t_{0} .
$$

First, we show by induction that

$$
\left\|\frac{I\left(2^{-n}\right)^{k} x-x}{k 2^{-n}}-y\right\| \leq \varepsilon \quad \text { for all } k, n \in \mathbb{N} \text { with } k 2^{-n} \leq t_{0} .
$$

For $k=1$, the claim holds by inequality (4.4). To prove the induction step, we assume for some fixed $k \in \mathbb{N}$ that

$$
\left\|\frac{I\left(2^{-n}\right)^{k} x-x}{k 2^{-n}}-y\right\| \leq \varepsilon \quad \text { for all } n \in \mathbb{N} \text { with } k 2^{-n} \leq t_{0} .
$$

Let $n \in \mathbb{N}$ with $(k+1) 2^{-n} \leq t_{0}$. It holds

$$
\begin{aligned}
\frac{I\left(2^{-n}\right)^{k+1} x-x}{(k+1) 2^{-n}}-y= & \frac{1}{k+1}\left(\frac{I\left(2^{-n}\right)^{k} I\left(2^{-n}\right) x-I\left(2^{-n}\right)^{k} x}{2^{-n}}-y\right) \\
& +\frac{k}{k+1}\left(\frac{I\left(2^{-n}\right)^{k} x-x}{k 2^{-n}}-y\right) .
\end{aligned}
$$

The first term is further decomposed as

$$
\begin{aligned}
\frac{I\left(2^{-n}\right)^{k} I\left(2^{-n}\right) x-I\left(2^{-n}\right)^{k} x}{2^{-n}-y=} & \frac{I\left(2^{-n}\right)^{k} I\left(2^{-n}\right) x-I\left(2^{-n}\right)^{k}\left(x+2^{-n} y\right)}{2^{-n}} \\
& +\frac{I\left(2^{-n}\right)^{k}\left(x+2^{-n} y\right)-I\left(2^{-n}\right)^{k} x}{2^{-n}}-y .
\end{aligned}
$$

We use Lemma 2.7 and inequality (4.4) to estimate

$$
\begin{aligned}
\left\|\frac{I\left(2^{-n}\right)^{k} I\left(2^{-n}\right) x-I\left(2^{-n}\right)^{k}\left(x+2^{-n} y\right)}{2^{-n}}\right\| & \leq e^{\omega_{\alpha(2 r, 1)}}\left\|\frac{I\left(2^{-n}\right) x-x}{2^{-n}}-y\right\| \\
& \leq e^{\omega_{\alpha(2 r, 1)}} \frac{\varepsilon}{2} e^{-\omega_{\alpha(2 r, 1)}}=\frac{\varepsilon}{2}
\end{aligned}
$$


Note that Lemma 2.7 relies only on Assumption 2.1, but not on Assumption 2.4. Combining inequality (4.5), equation (4.9) and inequality (4.10) yields

$$
\left\|\frac{I\left(2^{-n}\right)^{k} I\left(2^{-n}\right) x-I\left(2^{-n}\right)^{k} x}{2^{-n}}-y\right\| \leq \varepsilon .
$$

Furthermore, it follows from inequality (4.7), equation (4.8) and inequality (4.11) that

$$
\begin{aligned}
& \left\|\frac{I\left(2^{-n}\right)^{k+1} x-x}{(k+1) 2^{-n}}-y\right\| \\
& \leq \frac{1}{k+1}\left\|\frac{I\left(2^{-n}\right)^{k} I\left(2^{-n}\right) x-I\left(2^{-n}\right)^{k} x}{2^{-n}}-y\right\|+\frac{k}{k+1}\left\|\frac{I\left(2^{-n}\right)^{k} x-x}{k 2^{-n}}-y\right\| \leq \varepsilon .
\end{aligned}
$$

Second, we show that the right-hand side of equation (4.3) holds. Inequality (4.1) and inequality (4.6) imply

$$
\left\|\frac{S(t) x-x}{t}-y\right\| \leq \sup _{n \in \mathbb{N}}\left\|\frac{I\left(\pi_{n}^{t}\right) x-x}{t}-y\right\| \leq \varepsilon \quad \text { for all } t \in\left(0, t_{0}\right] \cap \mathcal{T} .
$$

Now, let $t \in\left(0, t_{0}\right]$ be arbitrary and choose a sequence $\left(t_{n}\right)_{n \in \mathbb{N}} \subset\left(0, t_{0}\right] \cap \mathcal{T}$ with $t_{n} \rightarrow t$. Since $(S(t))_{t \geq 0}$ is strongly continuous, we obtain

$$
\left\|\frac{S(t) x-x}{t}-y\right\|=\lim _{n \rightarrow \infty}\left\|\frac{S\left(t_{n}\right) x-x}{t_{n}}-y\right\| \leq \varepsilon .
$$

\subsection{Condition (4.2)}

If $I(t)$ is convex and monotone for all $t \geq 0$, inequality (4.2) is always satisfied. Furthermore, we will see in Section 6.5 an example, where $I(t)$ has none of these two properties.

Lemma 4.4 Let $X$ be a Banach lattice and $(I(t))_{t \geq 0}$ be a family of convex monotone operators $I(t): X \rightarrow X$, which satisfy Assumption 2.1 with $x_{0}:=0$. Furthermore, let $\mathcal{L}^{I} \subset X$ be dense. Then, condition (4.2) holds for all $x, y \in X$.

Proof We argue similar as in the proof of [24, Proposition 3.9]. Let $x, y \in X$ and $k, n \in \mathbb{N}$. Since the operator $I\left(2^{-n}\right)^{k}$ is convex, the mapping

$$
\mathbb{R} \rightarrow X, \lambda \mapsto I\left(2^{-n}\right)^{k}(x+\lambda y)-I\left(2^{-n}\right)^{k} x
$$

is convex and maps zero to zero. This implies

$$
\begin{aligned}
-I\left(2^{-n}\right)^{k}(x-y)+I\left(2^{-n}\right)^{k} x-y & \leq \frac{I\left(2^{-n}\right)^{k}(x+\lambda y)-I\left(2^{-n}\right)^{k} x}{\lambda}-y \\
& \leq I\left(2^{-n}\right)^{k}(x+y)-I\left(2^{-n}\right)^{k} x-y \text { for all } \lambda \in(0,1] .
\end{aligned}
$$

Hence, for $\lambda:=2^{-n}$, we obtain

$$
\begin{aligned}
& \left\|\frac{I\left(2^{-n}\right)^{k}\left(x+2^{-n} y\right)-I\left(2^{-n}\right)^{k} x}{2^{-n}}-y\right\| \\
& \leq\left\|I\left(2^{-n}\right)^{k}(x+y)-(x+y)\right\|+\left\|I\left(2^{-n}\right)^{k}(x-y)-(x-y)\right\|+\left\|I\left(2^{-n}\right)^{k} x-x\right\| .
\end{aligned}
$$

It remains to show

$$
\lim _{t \downarrow 0} \sup _{n \in \mathbb{N}}\left\|I\left(\pi_{n}^{t}\right) x-x\right\|=0 \quad \text { for all } x \in X .
$$


Let $x \in X$ and $\varepsilon>0$. We define $r:=\|x\|+1$ and choose $\delta \in(0,1]$ with

$$
\left(e^{\omega_{\alpha(r, 1)}}+1\right) \delta \leq \frac{\varepsilon}{2} .
$$

Since $\mathcal{L}^{I} \subset X$ is dense, there exists $y \in B(x, \delta) \cap \mathcal{L}^{I}$. By Lemma 2.8, there exists $c \geq 0$ with

Let $t_{0}>0$ such that

$$
\left\|I\left(\pi_{n}^{t}\right) y-y\right\| \leq c e^{t \omega_{\alpha(r, t)} t} \quad \text { for all } t \geq 0 .
$$

$$
c e^{t_{0} \omega_{\alpha\left(r, t_{0}\right)}} t_{0} \leq \frac{\varepsilon}{2} .
$$

We use Lemma 2.7, inequality (4.12), inequality (4.13) and the non-decreasingness of $\alpha$ in the second argument to conclude

$$
\begin{aligned}
\left\|I\left(\pi_{n}^{t}\right) x-x\right\| & \leq\left\|I\left(\pi_{n}^{t}\right) x-I\left(\pi_{n}^{t}\right) y\right\|+\|x-y\|+\left\|I\left(\pi_{n}^{t}\right) y-y\right\| \\
& \leq\left(e^{t \omega_{\alpha(r, t)}}+1\right)\|x-y\|+c e^{t \omega_{\alpha(r, t)}} t \leq \varepsilon
\end{aligned}
$$

for all $t \in\left[0, t_{0}\right] \cap \mathcal{T}$ and $n \in \mathbb{N}$ with $2^{n} t \in \mathbb{N}$. Note that Lemmas 2.7 and 2.8 rely only on Assumption 2.1, but not Assumption 2.4.

Remark 4.5 The previous result also holds if $I(t)$ is linear for all $t \geq 0$ without assuming the monotonicity, because linearity of $I\left(2^{-n}\right)^{k}$ implies

$$
\frac{I\left(2^{-n}\right)^{k}\left(x+2^{-n} y\right)-I\left(2^{-n}\right)^{k} x}{2^{-n}}-y=I\left(2^{-n}\right)^{k} y-y
$$

for all $x, y \in X$ and $k, n \in \mathbb{N}$.

\subsection{Invariance of the Domain and Uniqueness}

Let $(S(t))_{t \geq 0}$ be a strongly continuous semigroup. Furthermore, we assume that, for every $r, T \geq 0$, there exists $c \geq 0$ such that

$$
\|S(t) x-S(t) y\| \leq c\|x-y\| \quad \text { for all } t \in[0, T] \text { and } x, y \in B(0, r) .
$$

The local behaviour of the semigroup $(S(t))_{t \geq 0}$ is determined through the infinitesimal generator

$$
A: D(A) \rightarrow X, x \mapsto \lim _{t \downarrow 0} \frac{S(t) x-x}{t},
$$

where the domain $D(A)$ consists of all $x \in X$ for which the previous limit exists.

Lemma 4.6 For every $x \in D(A)$ and $t \geq 0$,

$$
S(t) x \in D(A) \Longleftrightarrow \lim _{h \downarrow 0} \frac{S(t)(x+h A x)-S(t) x}{h} \text { exists. }
$$

Proof Fix $x \in D(A)$ and $t \geq 0$. For every $h>0$,

$$
\frac{S(h) S(t) x-S(t) x}{h}=\frac{S(t) S(h) x-S(t)(x+h A x)}{h}+\frac{S(t)(x+h A x)-S(t) x}{h} .
$$

It follows from inequality (4.14) and the definition of the generator that

$$
\lim _{h \downarrow 0} \frac{S(t) S(h) x-S(t)(x+h A x)}{h}=0 .
$$

Hence, the equivalence Eq. (4.15) holds by definition of the generator. 
Remark 4.7 Let $X$ be a Banach lattice and $(S(t))_{t \geq 0}$ a semigroup of convex monotone operators. Then, the quotient on the right hand side of Eq. (4.15) is non-decreasing in $h>0$ and bounded from below. Hence, if the norm is order continuous, the limit on the right hand side of Eq. (4.15) exists, and the domain is invariant. For details, we refer to [14]. Typical examples are $L^{p}$-spaces and Orlicz hearts, see [35], while spaces of continuous functions with the supremum or $\kappa$-norm do not have this property. Thus, the domain of a nonlinear semigroup is in general not invariant, see [13] for a counter example. One possibility to overcome this problem is the extension of the semigroup to a space with order continuous norm, see [3]. Another one is to weaken the definition of the generator by using monotone convergence, see [13]. One can also rely on viscosity solutions, see [10, 12, 24]. Finally, we want to mention an upcoming paper, where we use $\Gamma$-convergence to study the generator on the symmetric Lipschitz set, which will be introduced in Section 5.

The same arguments as in [14, Theorem 3.5] lead to the following uniqueness result. For the sake of completeness, we provide a proof.

Theorem 4.8 Let $x \in X$ and $y: \mathbb{R}_{+} \rightarrow X$ be a continuous function with $y(0)=x$ and $y(t) \in D(A)$ for all $t \geq 0$. Furthermore, we assume that

$$
\lim _{h \downarrow 0} \frac{y(t+h)-y(t)}{h}=A y(t) \quad \text { for all } t \geq 0,
$$

where the existence of the limit is part of the assumption. Then, it holds $y(t)=S(t) x$ for all $t \geq 0$.

Proof We fix $t \geq 0$ and define $g:[0, t] \rightarrow X, s \mapsto S(t-s) y(s)$. First, we show

$$
\lim _{h \downarrow 0} \frac{g(s+h)-g(s)}{h}=0 \quad \text { for all } s \in[0, t] .
$$

For every $s \in[0, t]$ and $h \in(0, t-s]$,

$$
\frac{g(s+h)-g(s)}{h}=\frac{S(t-s-h) y(s+h)-S(t-s-h) S(h) y(s)}{h} .
$$

By assumption, it holds

$$
\frac{y(s+h)-S(h) y(s)}{h}=\frac{y(s+h)-y(s)}{h}-\frac{S(h) y(s)-y(s)}{h} \rightarrow 0 \quad \text { as } h \downarrow 0 .
$$

Hence, it follows from inequality (4.14) that

$$
\lim _{h \downarrow 0} \frac{g(s+h)-g(s)}{h}=0 .
$$

Second, we show that $g$ is continuous. We have already established that $g$ is right continuous, i.e., we only have to show left continuity. For every $s \in[0, t]$, continuity of $y$, strong continuity of $S$ and inequality (4.14) imply $y(s)=\lim _{h \downarrow 0} S(h) y(s-h)$. Hence, it follows from inequality (4.14) that

$$
g(s-h)-g(s)=S(t-s) S(h) y(s-h)-S(t-s) y(s) \rightarrow 0 \quad \text { as } h \downarrow 0 .
$$

Third, following the proof of [28, Lemma 1.1 in Section 2], one can show that every continuous function with vanishing right derivative is constant. In particular, we obtain $y(t)=g(t)=g(0)=S(t) x$. 
Remark 4.9 If the domain is invariant and dense, the semigroup $(S(t))_{t \geq 0}$ is uniquely determined through its generator. In general, we do not know if the left derivative of the function $y$ in the previous theorem exists and the abstract Cauchy problem

$$
y^{\prime}(t)=A y(t) \quad \text { for all } t \geq 0, \quad y(0)=x,
$$

has classical solution. But we know that there exists at most one solution, and if the solution exists it depends locally Lipschitz continuous on the initial value $x$. If the norm is order continuous, the existence of a solution is also known, see [14].

\section{Symmetric Lipschitz Set}

Throughout this section, let $(S(t))_{t \geq 0}$ and $\left(S^{ \pm}(t)\right)_{t \geq 0}$ be three semigroups on a Banach lattice $X$. If we choose $S^{+}(t):=S(t)$ and $S^{-}(t) x:=-S(t)(-x)$ for all $t \geq 0$ and $x \in X$, the set

$$
\mathcal{L}_{\text {sym }}^{S}:=\mathcal{L}^{S^{+}} \cap \mathcal{L}^{S^{-}}=\left\{x \in \mathcal{L}^{S}:-x \in \mathcal{L}^{S}\right\}
$$

is called symmetric Lipschitz set of $(S(t))_{t \geq 0}$. In examples, this set can be determined explicitly, see Section 6.1. Furthermore, by defining the generator w.r.t. a weaker norm or pointwise almost everywhere, for elements of the symmetric Lipschitz set, the generator can be determined explicitly, see [3, 13]. In order to solve the corresponding Cauchy problem for all positive times, it is crucial to show that the symmetric Lipschitz set is invariant under the semigroup. Compared to the invariance of the domain, the invariance of the symmetric Lipschitz set does not depend on the underlying space, i.e., it also holds for spaces of continuous function.

Theorem 5.1 Assume that, for all $s, t \geq 0$ and $x \in X$,

- $S^{-}(t) x \leq S(t) x \leq S^{+}(t) x$,

- $S(s) S^{-}(t) x \leq S^{-}(t) S(s) x$ and $S^{+}(s) S(t) x \leq S(t) S^{+}(s) x$.

Furthermore, for every $r, T \geq 0$, there exists $c \geq 0$ such that, for all $t \in[0, T]$ and $x, y \in B(0, r)$,

$$
\|S(t) x-S(t) y\| \leq c\|x-y\| \quad \text { and } \quad\left\|S^{ \pm}(t) x-S^{ \pm}(t) y\right\| \leq c\|x-y\| .
$$

Then, it holds $\mathcal{L}^{S^{+}} \cap \mathcal{L}^{S^{-}} \subset \mathcal{L}^{S}$ and $S(t): \mathcal{L}^{S^{+}} \cap \mathcal{L}^{S^{-}} \rightarrow \mathcal{L}^{S^{+}} \cap \mathcal{L}^{S^{-}}$for all $t \geq 0$.

Proof First, we show $\mathcal{L}^{S^{+}} \cap \mathcal{L}^{S^{-}} \subset \mathcal{L}^{S}$. Let $x \in \mathcal{L}^{S^{+}} \cap \mathcal{L}^{S^{-}}$. By definition, there exist $t_{0}>0$ and $c \geq 0$ such that

$$
\left\|S^{ \pm}(t) x-x\right\| \leq c t \quad \text { for all } t \in\left[0, t_{0}\right] .
$$

Furthermore, by assumption, it holds

$$
S^{-}(t) x-x \leq S(t) x-x \leq S^{+}(t) x-x \text { for all } t \geq 0 .
$$

We obtain the estimate

$$
\|S(t) x-x\| \leq \max \left\{\left\|S^{+}(t) x-x\right\|,\left\|S^{-}(t) x-x\right\|\right\} \leq c t \quad \text { for all } t \in\left[0, t_{0}\right] .
$$

Second, we show $S(t): \mathcal{L}^{S^{+}} \cap \mathcal{L}^{S^{-}} \rightarrow \mathcal{L}^{S^{+}} \cap \mathcal{L}^{S^{-}}$for all $t \geq 0$. Let $x \in \mathcal{L}^{S^{+}} \cap \mathcal{L}^{S^{-}}$and $t \geq 0$. By definition and the first part, there exist $t_{0}>0$ and $c \geq 0$ such that

$$
\|S(t) x-x\| \leq c t \quad \text { and } \quad\left\|S^{ \pm}(t) x-x\right\| \leq c t \quad \text { for all } t \in\left[0, t_{0}\right] .
$$


It follows from the assumptions of the theorem that, for all $s, t \geq 0$,

$$
\begin{aligned}
S(s) S^{-}(t) x-S(s) x & \leq S^{-}(t) S(s) x-S(s) x \leq S(t) S(s) x-S(s) x, \\
S(t) S(s) x-S(s) x & \leq S^{+}(t) S(s) x-S(s) x \leq S(s) S^{+}(t) x-S(s) x .
\end{aligned}
$$

We use inequality (5.1) and inequality (5.2) to estimate

$$
\begin{aligned}
\left\|S^{-}(t) S(s) x-S(s) x\right\| & \leq \max \left\{\|S(s) S(t) x-S(s) x\|,\left\|S(s) S^{-}(t) x-S(s) x\right\|\right\} \\
& \leq c_{1} \max \left\{\|S(t) x-x\|,\left\|S^{-}(t) x-x\right\|\right\} \leq c c_{1} t, \\
\left\|S^{+}(t) S(s) x-S(s) x\right\| & \leq \max \left\{\|S(s) S(t) x-S(s) x\|,\left\|S(s) S^{+}(t) x-S(s) x\right\|\right\} \\
& \leq c_{1} \max \left\{\|S(t) x-x\|,\left\|S^{+}(t) x-x\right\|\right\} \leq c c_{1} t
\end{aligned}
$$

for all $s \in\left[0, t_{0}\right]$ and $t \geq 0$. Here, $c_{1}$ is a constant such that inequality (5.1) holds with $T:=t_{0}$ and $r:=\max \left\{\|S(t) x\|,\left\|S^{ \pm}(t) x\right\|\right\}$.

The following assumption on the families $(I(t))_{t \geq 0}$ and $\left(I^{ \pm}(t)\right)_{t \geq 0}$ ensures that the associated semigroups $(S(t))_{t \geq 0}$ and $\left(S^{ \pm}(t)\right)_{t \geq 0}$ satisfy the assumptions of Theorem 5.1. Furthermore, they allow us to study the relation between the Lipschitz sets of the generating families and the associated semigroups.

Assumption 5.2 Let $(I(t))_{t \geq 0}$ and $\left(I^{ \pm}(t)\right)_{t \geq 0}$ be three families of monotone operators on $X$, which satisfy Assumption 2.1 with $x_{0}:=0$. We assume, for all $s, t \geq 0$ and $x \in X$,

(i) $I^{-}(t) x \leq I(t) x \leq I^{+}(t) x$,

(ii) $I(s) I^{-}(t) x \leq I^{-}(t) I(s) x$ and $I^{+}(s) I(t) x \leq I(t) I^{+}(s) x$,

(iii) $I(s+t) x \leq I(s) I(t)$,

(iv) $I^{+}(t)$ is continuous from below.

In addition, we assume, for all $(x, t) \in X \times \mathbb{R}_{+}$,

$$
S(t) x=\sup _{\pi \in P_{t}} I(\pi) x, \quad S^{+}(t) x=\sup _{\pi \in P_{t}} I^{+}(\pi) x, \quad \text { and } \quad S^{-}(t)=\inf _{\pi \in P_{t}} I^{-}(\pi) x .
$$

Theorem 5.3 Suppose that Assumption 5.2 is satisfied. Then,

$$
\mathcal{L}^{I^{+}} \cap \mathcal{L}^{I^{-}}=\mathcal{L}^{S^{+}} \cap \mathcal{L}^{S^{-}} .
$$

Furthermore, it holds $S(t): \mathcal{L}^{S^{+}} \cap \mathcal{L}^{S^{-}} \rightarrow \mathcal{L}^{S^{+}} \cap \mathcal{L}^{S^{-}}$for all $t \geq 0$.

Proof W.l.o.g. we assume that $(I(t))_{t \geq 0}$ and $\left(I^{ \pm}(t)\right)_{t \geq 0}$ satisfy Assumption 2.1 with the same function $\alpha$ and the same constant $\omega_{r}$ for all $r \geq 0$. First, we show that $\mathcal{L}^{I^{+}} \cap \mathcal{L}^{I^{-}}=$ $\mathcal{L}^{S^{+}} \cap \mathcal{L}^{S^{-}}$. Let $x \in \mathcal{L}^{I^{+}} \cap \mathcal{L}^{I^{-}}$. By definition, there exist $t_{0}>0$ and $c \geq 0$ such that

$$
\left\|I^{ \pm}(t) x-x\right\| \leq c t \quad \text { for all } t \in\left[0, t_{0}\right] .
$$

It follows from Assumption 5.2 and Remark 2.14(ii) that

$$
\left\|S^{ \pm}(t) x-x\right\| \leq \sup _{\pi \in P_{t}}\left\|I^{ \pm}(\pi) x-x\right\| \leq c e^{t \omega_{\alpha(r, t)} t \quad \text { for all } t \geq 0,}
$$

where $r:=\|x\|$. On the other hand, let $x \in \mathcal{L}^{S^{+}} \cap \mathcal{L}^{S^{-}}$. By definition, there exist $t_{0}>0$ and $c \geq 0$ such that

$$
\left\|S^{ \pm}(t) x-x\right\| \leq c t \quad \text { for all } t \in\left[0, t_{0}\right] .
$$

Assumption 5.2 implies

$$
S^{-}(t) x-x \leq I^{-}(t) x-x \leq I^{+}(t) x-x \leq S^{+}(t) x-x,
$$


and therefore

$$
\left\|I^{ \pm}(t) x-x\right\| \leq \max \left\{\left\|S^{+}(t) x-x\right\|,\left\|S^{-}(t) x-x\right\|\right\} \leq c t \quad \text { for all } t \in\left[0, t_{0}\right] .
$$

Second, we show $S^{-}(t) x \leq S(t) x \leq S^{+}(t) x$ for all $(x, t) \in X \times \mathbb{R}_{+}$. It follows immediately from Assumption 5.2 that $S^{-}(t) x \leq I^{-}(t) x \leq I(t) x \leq S(t) x$. Furthermore, Assumption 5.2(i) and the monotonicity of $I(s)$ imply

$$
I(s) I(t) x \leq I(s) I^{+}(t) x \leq I^{+}(s) I^{+}(t) x \text { for all } s, t \geq 0 \text { and } x \in X .
$$

By Assumption 5.2 and induction, we obtain

$$
S(t) x=\sup _{\pi \in P_{t}} I(\pi) x \leq \sup _{\pi \in P_{t}} I^{+}(\pi) x=S^{+}(t) x \quad \text { for all }(x, t) \in X \times \mathbb{R}_{+} .
$$

Third, we show $S(s) S^{-}(t) x \leq S^{-}(t) S(s) x$ and $S^{+}(s) S(t) x \leq S(t) S^{+}(s) x$ for all $s, t \geq$ 0 and $x \in X$. We use Assumption 5.2(ii) and the monotonicity of $(I(t))_{t \geq 0}$ and $\left(I^{-}(t)\right)_{t \geq 0}$ to estimate

$$
I\left(s_{1}\right) I\left(s_{2}\right) I^{-}\left(t_{1}\right) I^{-}\left(t_{2}\right) \leq I^{-}\left(t_{1}\right) I^{-}\left(t_{2}\right) I\left(s_{1}\right) I\left(s_{2}\right)
$$

for all $s_{1}, s_{2}, t_{1}, t_{2} \geq 0$. It follows by induction that

$$
I\left(\pi_{s}\right) I^{-}\left(\pi_{t}\right) x \leq I^{-}\left(\pi_{t}\right) I\left(\pi_{s}\right) x
$$

for all $s, t \geq 0, \pi_{s} \in P_{s}, \pi_{t} \in P_{t}$ and $x \in X$. Hence, Assumption 5.2 and the monotonicity of $I\left(\pi_{s}\right)$ and $I^{-}\left(\pi_{t}\right)$ imply

$$
\begin{aligned}
S(s) S^{-}(t) x & =\sup _{\pi_{s} \in P_{s}} I\left(\pi_{s}\right)\left(\inf _{\pi_{t} \in P_{t}} I^{-}\left(\pi_{t}\right) x\right) \leq \sup _{\pi_{s} \in P_{s}} \inf _{\pi_{t} \in P_{t}} I\left(\pi_{s}\right) I^{-}\left(\pi_{t}\right) x \\
& \leq \sup _{\pi_{s} \in P_{s}} \inf _{\pi_{t} \in P_{t}} I^{-}\left(\pi_{t}\right) I\left(\pi_{s}\right) x \leq \inf _{\pi_{t} \in P_{t}} \sup _{\pi_{s} \in P_{s}} I^{-}\left(\pi_{t}\right) I\left(\pi_{s}\right) x \\
& \leq \inf _{\pi_{t} \in P_{t}} I^{-}\left(\pi_{t}\right)\left(\sup _{\pi_{s} \in P_{S}} I\left(\pi_{s}\right)\right)=S^{-}(t) S(s) x .
\end{aligned}
$$

Similarly, we use Assumption 5.2(ii) and the monotonicity of $(I(t))_{t \geq 0}$ and $\left(I^{+}(t)\right)_{t \geq 0}$ to obtain

$$
I^{+}\left(\pi_{s}\right) I\left(\pi_{t}\right) x \leq I\left(\pi_{t}\right) I^{+}\left(\pi_{s}\right) x
$$

for all $s, t \geq 0, \pi_{s} \in P_{s}, \pi_{t} \in P_{t}$ and $x \in X$. Moreover, Assumption 5.2(iii) implies that the set $\left\{I(\pi): \pi \in P_{t}\right\}$ is directed upwards for all $t \geq 0$, and Assumption 5.2(iv) ensures that $I^{+}(\pi)$ is continuous from below for all $s \geq 0$ and $\pi \in P_{s}$. We combine this with the monotonicity of $I\left(\pi_{t}\right)$ to conclude

$$
\begin{aligned}
S^{+}(s) S(t) & =\sup _{\pi_{s} \in P_{s}} I^{+}\left(\pi_{s}\right)\left(\sup _{\pi_{t} \in P_{t}} I\left(\pi_{t}\right) x\right)=\sup _{\pi_{s} \in P_{s}} \sup _{\pi_{t} \in P_{t}} I^{+}\left(\pi_{s}\right) I\left(\pi_{t}\right) x \\
& \leq \sup _{\pi_{t} \in P_{t}} \sup _{\pi_{s} \in P_{s}} I\left(\pi_{t}\right) I^{+}\left(\pi_{s}\right) x \leq \sup _{\pi_{t} \in P_{t}} I\left(\pi_{t}\right)\left(\sup _{\pi_{s} \in P_{s}} I^{+}\left(\pi_{s}\right) x\right)=S(t) S^{+}(s) x
\end{aligned}
$$

for all $s, t \geq 0$ and $x \in X$.

Fifth, inequality (5.1) follows from Assumption 2.1 and Remark 2.14(i). Hence, we can apply Theorem 5.1 and obtain $S(t): \mathcal{L}^{S^{+}} \cap \mathcal{L}^{S^{-}} \rightarrow \mathcal{L}^{S^{+}} \cap \mathcal{L}^{S^{-}}$for all $t \geq 0$.

Remark 5.4 Let $(I(t))_{t \geq 0}$ be a family of operators $I(t): X \rightarrow X$, which satisfy Assumption 2.1 with $x_{0}:=0$ and Assumption 2.4. We define $I^{+}(t):=I(t)$ and $I^{-}(t) x:=$ $-I(t)(-x)$ for all $(x, t) \in X \times \mathbb{R}_{+}$. Then, the families $\left(I^{ \pm}(t)\right)_{t \geq 0}$ satisfy Assumption 2.1 with $x_{0}=0$. Furthermore, Assumption 2.4 is satisfied, for example, if the set $\mathcal{D}$ can be chosen symmetric, i.e. $\mathcal{D}=\{-x: x \in \mathcal{D}\}$. Let $(S(t))_{t \geq 0}$ and $\left(S^{ \pm}(t)\right)_{t \geq 0}$ be associated semigroups as in Theorem 2.5. Then, by construction, it holds $S^{+}(t)=S(t)$ and 
$S^{-}(t) x=-S(t)(-x)$ for all $(x, t) \in X \times \mathbb{R}_{+}$. The condition $S(t) x=\sup _{\pi \in P_{t}} I(\pi) x$ has already been discussed in Section 2.2. In that case, we conclude

$$
S^{-}(t) x=-S(t)(-x)=-\sup _{\pi \in P_{t}} I(\pi)(-x)=\inf _{\pi \in P_{t}}-I(\pi)(-x)=\inf _{\pi \in P_{t}} I^{-}(\pi)(x) .
$$

We will see in Section 6.1 that the verification of Assumption 5.2(i)-(iv) is straightforward, if $I(t)$ is a supremum over linear semigroups. Basically, condition (ii) is the consequence of interchanging a supremum with an infimum at the cost of an inequality. Furthermore, it is clear that Theorem 5.3 remains true without Assumption 5.2(iii) and (iv), if $I(t)=I^{+}(t)$ for all $t \geq 0$.

\section{Examples}

We illustrate our main results with several examples. First, we consider Nisio semigroups, which have already been discussed in Section 2.2. This first type of examples is illustrated by a convex version of the $g$-expectation as well as a sublinear version the geometric Brownian motion and the $G$-expectation. Second, we start with a linear semigroup $\left(S_{0}(t)\right)_{t \geq 0}$ and consider the generating family $(I(t))_{t \geq 0}$ given as the perturbation

$$
I(t) x:=S_{0}(t) x+t \Psi(x) \text { for all }(x, t) \in X \times \mathbb{R}_{+},
$$

where $\Psi: X \rightarrow X$ is a Lipschitz continuous mapping. If we choose $X:=\mathbb{R}^{d}$ and $S_{0}(t):=$ $\mathrm{id}_{\mathbb{R}^{d}}$, for every $x \in \mathbb{R}^{d}$, we obtain the unique solution to the ODE

$$
y^{\prime}(t)=f(y(t)) \quad \text { for all } t \geq 0, \quad y(0)=x .
$$

The main example of this second type are reaction-diffusion equations, where the operators $I(t)$ are neither convex nor monotone. Recall that the results in Section 2 do not rely on these properties. The verification of condition (4.2) becomes more complicated, but is possible by proving a suitable recursion formula for the iterated operators $I\left(2^{-n}\right)^{k}$.

The theory and the examples in this paper are independent of results from established PDE theory. For a common approach to nonlinear parabolic equations we refer to [23]. There, short time existence is proved by a fixed-point argument, and, as long as the solution does not blow up, long time existence follows. In the semi-linear case, blow up in finite time is excluded, if the non-linearity is locally Lipschitz continuous and does not grow faster than linear. While the approach in [23] relies strongly on a priori estimates in suitable function spaces, we use stochastic representations for the generating family $(I(t))_{t \geq 0}$ and Itô calculus.

\subsection{Convex G-expectation}

In this subsection, we construct a semigroup, which corresponds to a Brownian motion with uncertain drift. The generator is a semi-linear second order differential operator, where the first-order non-linearity corresponds to the uncertainty in the drift. Let $\left(W_{t}\right)_{t \geq 0}$ be a $d$ dimensional Brownian motion on a probability space $(\Omega, \mathcal{F}, \mathbb{P})$. Furthermore, let $L: \mathbb{R}^{d} \rightarrow$ $[0, \infty]$ be a function such that

- $\min _{\lambda \in \mathbb{R}^{d}} L(\lambda)=0$,

- $\lim _{|\lambda| \rightarrow \infty} L(\lambda) /|\lambda|=\infty$. 
For every $t \geq 0, f \in C_{0}\left(\mathbb{R}^{d} ; \mathbb{R}\right)$ and $x \in \mathbb{R}^{d}$, we define

$$
(I(t) f)(x):=\sup _{\lambda \in \mathbb{R}^{d}}\left(\mathbb{E}\left[f\left(x+W_{t}+\lambda t\right)\right]-L(\lambda) t\right),
$$

where $\mathbb{E}[X]$ denotes the expectation of random variable $X: \Omega \rightarrow \mathbb{R}$. For every $\lambda \in \mathbb{R}^{d}$, we denote by $\left(S_{\lambda}(t)\right)_{t \geq 0}$ the linear semigroup given by

$$
\left(S_{\lambda}(t) f\right)(x):=\mathbb{E}\left[f\left(x+W_{t}+\lambda t\right)\right] \text { for all } t \geq 0, f \in C_{0} \text { and } x \in \mathbb{R}^{d} .
$$

Moreover, we can write $I(t) f=\sup _{\lambda \in \mathbb{R}^{d}} I_{\lambda}(t) f$ for all $t \geq 0$ and $f \in C_{0}$ by defining $I_{\lambda}(t) f:=S_{\lambda}(t) f-L(\lambda) t$ for all $\lambda \in \mathbb{R}^{d}$. The first-order non-linearity will be described by the function

$$
H: \mathbb{R}^{d} \rightarrow \mathbb{R}, x \mapsto \sup _{y \in \mathbb{R}^{d}}(\langle x, y\rangle-L(y)),
$$

where $\langle\cdot, \cdot\rangle$ denotes the Euclidean inner product on $\mathbb{R}^{d}$. Note that we are not restricted to non-linearities with linear growth. For example, power functions $H(x):=|x|^{p}$ with $p \geq 1$ are included in our setting.

Lemma 6.1 For every $c \geq 0$, there exists a bounded set $\Lambda \subset \mathbb{R}^{d}$ such that

$$
I(t) f=\sup _{\lambda \in \Lambda} I_{\lambda}(t) f \quad \text { for all } t \geq 0 \text { and } f \in \operatorname{Lip}_{0}(c) .
$$

Proof Let $c \geq 0, f \in \operatorname{Lip}_{0}(c)$ and $\lambda_{0} \in \mathbb{R}^{d}$ with $L\left(\lambda_{0}\right)<\infty$, which exists by assumption. For every $\lambda \in \mathbb{R}^{d}, t \geq 0$ and $x \in \mathbb{R}^{d}$,

$$
\begin{aligned}
\mathbb{E}\left[f\left(x+W_{t}+\lambda t\right)\right]-L(\lambda) t & \leq \mathbb{E}\left[f\left(x+W_{t}+\lambda_{0} t\right)\right]+c\left|\lambda-\lambda_{0}\right| t-L(\lambda) t \\
& =\left(I_{\lambda_{0}}(t) f\right)(x)+\left(L\left(\lambda_{0}\right)+c\left|\lambda-\lambda_{0}\right|-L(\lambda)\right) t .
\end{aligned}
$$

Since the assumption on $L$ implies $L\left(\lambda_{0}\right)+c\left|\lambda-\lambda_{0}\right|-L(\lambda) \rightarrow-\infty$ as $|\lambda| \rightarrow \infty$, the claim follows.

Let $C_{0}^{2}$ be the space of all twice continuously differentiable functions $f \in C_{0}$ such that the first and second derivative vanish at infinity. For every $\lambda \in \mathbb{R}^{d}$, we denote by $A_{\lambda}$ the generator of $\left(S_{\lambda}(t)\right)_{t \geq 0}$. It follows from Itô's formula that $C_{0}^{2} \subset D\left(A_{\lambda}\right)$ with

$$
A_{\lambda} f=\frac{1}{2} \Delta f+\nabla_{\lambda} f \quad \text { for all } f \in C_{0}^{2}, \text { where } \nabla_{\lambda} f:=\langle\lambda, \nabla f\rangle .
$$

Theorem 6.2 The family $(I(t))_{t \geq 0}$ satisfies the assumptions of Lemma 3.2. Hence, Theorem 2.5 yields an associated semigroup $(S(t))_{t \geq 0}$ on $C_{0}$. Moreover, it holds $C_{0}^{2} \subset D(A)$ with

$$
A f=\sup _{\lambda \in \mathbb{R}^{d}} A_{\lambda} f=\frac{1}{2} \Delta f+H(\nabla f) \quad \text { for all } f \in C_{0}^{2} .
$$

Proof First, we verify the conditions (i)-(iv) of Lemma 3.2.

1. Clearly, $I(0)=\mathrm{id}_{C_{0}}$.

2. For every $t \geq 0$ and $f \in C_{0}$, the first assumption on $L$ implies

$$
-\|f\|_{\infty}=-\|f\|_{\infty}-\inf _{\lambda \in \mathbb{R}^{d}} L(\lambda) t \leq I(t) f \leq \sup _{\lambda \in \mathbb{R}^{d}} S_{\lambda}(t) f \leq\|f\|_{\infty} .
$$

We obtain $\|I(t) f\|_{\infty} \leq\|f\|_{\infty}$. 
3. For every $\lambda \in \mathbb{R}^{d}, t \geq 0$ and $f, g \in C_{0}$,

$$
I_{\lambda}(t) f-I(t) g \leq I_{\lambda}(t) f-I_{\lambda}(t) g=S_{\lambda}(t)(f-g) \leq\|f-g\|_{\infty} .
$$

By taking the supremum over $\lambda \in \mathbb{R}^{d}$ and changing the role of $f$ and $g$, we obtain $\|I(t) f-I(t) g\|_{\infty} \leq\|f-g\|_{\infty}$.

4. For every $t \geq 0, f \in C_{0}$ and $x \in \mathbb{R}^{d}$,

$$
\left(I(t) f_{x}\right)(0)=\sup _{\lambda \in \mathbb{R}^{d}}\left(\mathbb{E}\left[f\left(x+\left(0+W_{t}+\lambda t\right)\right)\right]-L(\lambda) t\right)=(I(t) f)(x) .
$$

Second, we show that

$$
\lim _{t \downarrow 0}\left\|\frac{I(t) f-f}{t}-\frac{1}{2} \Delta f-H(\nabla f)\right\|_{\infty}=0 \quad \text { for all } f \in C_{0}^{2} .
$$

Let $f \in C_{0}^{2}$. By Lemma 6.1, there exists $r \geq 0$ such that

$$
I(t) f=\sup _{\lambda \in B(0, r)} I_{\lambda}(t) f \quad \text { for all } t \geq 0 .
$$

Moreover, the constant $r \geq 0$ can be chosen such that

$$
H(\nabla f)=\sup _{\lambda \in B(0, r)}(\langle\lambda, \nabla f\rangle-L(\lambda)),
$$

because $\|\nabla f\|_{\infty}<\infty$ and $L$ growths faster than linear. Hence, it follows from Itô's formula that

$$
\begin{aligned}
& \left\|\frac{I(t) f-f}{t}-\frac{1}{2} \Delta f-H(\nabla f)\right\|_{\infty} \leq \sup _{\lambda \in B(0, r)}\left\|\frac{S_{\lambda}(t) f-f}{t}-\frac{1}{2} \Delta f-\langle\lambda, \nabla f\rangle\right\|_{\infty} \\
& \leq \sup _{\lambda \in B(0, r)} \frac{1}{t} \int_{0}^{t}\left(\left\|\nabla_{\lambda} f\left(\cdot+X_{s}^{\lambda}\right)-\nabla_{\lambda} f\right\|_{\infty}+\frac{1}{2}\left\|\Delta f\left(\cdot+X_{s}^{\lambda}\right)-\Delta f\right\|_{\infty}\right) \mathrm{d} s,
\end{aligned}
$$

where $X_{s}^{\lambda}:=W_{s}+\lambda s$ for all $\lambda \in \mathbb{R}^{d}$ and $s \geq 0$. Since $f \in C_{0}^{2}$, for every $\varepsilon>0$, there exists $\delta>0$ such that

$$
\left(\left\|\nabla_{\lambda} f\left(\cdot+X_{s}^{\lambda}\right)-\nabla_{\lambda} f\right\|_{\infty}+\frac{1}{2}\left\|\Delta f\left(\cdot+X_{s}^{\lambda}\right)-\Delta f\right\|_{\infty}\right) \mathbb{1}_{\left\{\left|X_{s}^{\lambda}\right|<\delta\right\}}<\varepsilon
$$

for all $\lambda \in \mathbb{R}^{d}$ and $s \geq 0$. Furthermore, Chebyshev's inequality implies

$$
\sup _{\lambda \in B(0, r)} \mathbb{P}\left(\left|X_{s}^{\lambda}\right| \geq \delta\right) \leq \sup _{\lambda \in B(0, r)} \frac{\mathbb{E}\left[\left|X_{s}^{\lambda}\right|^{2}\right]}{\delta^{2}} \leq \sup _{\lambda \in B(0, r)} \frac{2}{\delta^{2}}\left(\mathbb{E}\left[\left|W_{s}\right|^{2}\right]+|\lambda|^{2} s^{2}\right) \rightarrow 0 \quad \text { as } s \downarrow 0 .
$$

Third, we verify the conditions (v) and (vi) of Lemma 3.2. It follows immediately from inequality (6.1) that $C_{0}^{2} \subset \operatorname{Lip}_{0} \cap \mathcal{L}^{I}$. In particular, we can choose a countable set $\mathcal{D} \subset$ $\operatorname{Lip}_{0} \cap \mathcal{L}^{I}$, which is dense in $C_{0}$. It remains to show condition (vi). Let $c \geq 0$ and choose $r \geq 0$ such that

$$
I(t) f=\sup _{\lambda \in B(0, r)} I_{\lambda}(t) f \quad \text { for all } t \geq 0 \text { and } f \in \operatorname{Lip}_{0}(c),
$$

which exists due to Lemma 6.1. We define

$$
\left(T_{c}(t) f\right)(x):=e^{\frac{r^{2} t}{2}} \mathbb{E}\left[f^{2}\left(x+W_{t}\right)\right]^{\frac{1}{2}} \quad \text { for all } t \geq 0, f \in C_{0}^{+} \text {and } x \in \mathbb{R}^{d} .
$$

The family $\left(T_{c}(t)\right)_{t \geq 0}$ is a monotone semigroup on $C_{0}^{+}$. It remains to show

$$
|I(t) f| \leq T_{c}(t)|f| \text { for all } f \in \operatorname{Lip}_{0}(c) \text { and } t \geq 0 .
$$


Let $f \in \operatorname{Lip}_{0}(c)$ and $t \geq 0$. We use $W_{t} \sim \mathcal{N}(0, t \mathbb{1})$, where $\mathcal{N}(0, t \mathbb{1})$ denotes the normal distribution, and the formula for its moment generating function to estimate

$$
\begin{aligned}
& \left|\left(S_{\lambda}(t) f\right)(x)\right| \leq \mathbb{E}\left[\left|f\left(x+W_{t}+\lambda t\right)\right|\right] \\
& =(2 \pi t)^{-\frac{d}{2}} \int_{\mathbb{R}^{d}}|f(x+y+\lambda t)| \exp \left(-\frac{|y|^{2}}{2 t}\right) \mathrm{d} y \\
& =(2 \pi t)^{-\frac{d}{2}} \int_{\mathbb{R}^{d}}|f(x+y)| \exp \left(-\frac{|y-\lambda t|^{2}}{2 t}\right) \mathrm{d} y \\
& =e^{-\frac{|\lambda|^{2} t}{2}} \int_{\mathbb{R}^{d}}|f(x+y)| \exp (\langle\lambda, y\rangle) \mathcal{N}(0, t \mathbb{1})(\mathrm{d} y) \\
& \leq e^{-\frac{|\lambda|^{2} t}{2}}\left(\int_{\mathbb{R}^{d}} f^{2}(x+y) \mathcal{N}(0, t \mathbb{1})(\mathrm{d} y)\right)^{\frac{1}{2}}\left(\int_{\mathbb{R}^{d}} \exp (2\langle\lambda, y\rangle) \mathcal{N}(0, t \mathbb{1})(\mathrm{d} y)\right)^{\frac{1}{2}} \\
& =e^{-\frac{|\lambda|^{2} t}{2}} \mathbb{E}\left[f^{2}\left(x+W_{t}\right)\right]^{\frac{1}{2}} e^{|\lambda|^{2} t}=e^{\frac{|\lambda|^{2} t}{2}} \mathbb{E}\left[f^{2}\left(x+W_{t}\right)\right]^{\frac{1}{2}} \leq\left(T_{c}(t)|f|\right)(x)
\end{aligned}
$$

for all $\lambda \in B(0, r)$. Taking the supremum yields

$$
I(t) f \leq \sup _{\lambda \in B(0, r)} S_{\lambda}(t) f \leq T_{c}(t)|f| .
$$

Furthermore, by assumption, there exists $\lambda_{0} \in \mathbb{R}^{d}$ with $L\left(\lambda_{0}\right)=0$. W.l.o.g. we can assume $r \geq\left|\lambda_{0}\right|$ and obtain $I(t) f \geq S_{\lambda_{0}}(t) f \geq-T_{c}(t)|f|$.

Forth, by Theorem 2.5, there exists an associated semigroup $(S(t))_{t \geq 0}$ on $C_{0}$. In particular, Assumption 4.1 is satisfied. In addition, it follows from Lemma 4.4 that condition (4.2) holds for all $f, g \in C_{0}$. Hence, Theorem 4.3 implies $C_{0}^{2} \subset D(A)$ with

$$
\text { Af }=\frac{1}{2} \Delta f+H(\nabla f) \text { for all } f \in C_{0}^{2} .
$$

Let $L^{\infty}$ be the space of all bounded, Borel measurable functions $f: \mathbb{R}^{d} \rightarrow \mathbb{R}$, where two of them are identified if they coincide Lebesgue almost everywhere. Moreover, we denote by $W^{1, \infty}$ the corresponding first order Sobolev space. For $f \in W^{1, \infty}$ we say that $\Delta f$ exists in $L^{\infty}$ if there exists a function $g \in L^{\infty}$ with

$$
\int_{\mathbb{R}^{d}} g \phi \mathrm{d} x=-\int_{\mathbb{R}^{d}}\langle\nabla f, \nabla \phi\rangle \mathrm{d} x \quad \text { for all } \phi \in C_{c}^{\infty} .
$$

In this case, since $g$ is unique Lebesgue almost everywhere, we define $\Delta f:=g$. Here, $C_{c}^{\infty}$ denotes the set of all infinitely differentiable functions $\phi: \mathbb{R}^{d} \rightarrow \mathbb{R}$ with compact support.

Theorem 6.3 In addition to previous assumptions, we assume that L satisfies

- $\sup _{\lambda \in B(0, r) \cap\{L<\infty\}} L(\lambda)<\infty$ for all $r>0$,

- there exists $\varepsilon>0$ with $\sup _{\{|\lambda|=\varepsilon\}} L(\lambda)<\infty$.

Then, it holds $S(t): \mathcal{L}_{\text {sym }}^{S} \rightarrow \mathcal{L}_{\text {sym }}^{S}$ for all $t \geq 0$, and

$$
\mathcal{L}_{\text {sym }}^{S}=\mathcal{L}_{\text {sym }}^{I}=\left\{f \in W^{1, \infty} \cap C_{0}: \Delta f \text { exists in } L^{\infty}\right\} .
$$

Proof We define $I^{+}(t):=I(t)$ and $I^{-}(t) x:=-I(t)(-x)$ for all $t \geq 0$ and $x \in X$. First, we verify the conditions (i)-(iii) of Assumption 5.2. 
1. It holds $I^{-}(t) f=-I(t)(-f) \leq I(t) f=I^{+}(t) f$ for all $(f, t) \in C_{0} \times \mathbb{R}_{+}$, because $I(t)$ is convex and $I(t) 0=0$.

2. Let $s, t \geq 0$ and $f \in C_{0}$. We use Fubini's theorem to conclude

$$
\begin{aligned}
I(s) I^{-}(t) f & =\sup _{\lambda_{1} \in \mathbb{R}^{d}}\left(S_{\lambda_{1}}(s)\left(\inf _{\lambda_{2} \in \mathbb{R}^{d}}\left(S_{\lambda_{2}}(t) f+L\left(\lambda_{2}\right) t\right)\right)-L\left(\lambda_{1}\right) s\right) \\
& \leq \sup _{\lambda_{1} \in \mathbb{R}^{d}} \inf _{\lambda_{2} \in \mathbb{R}^{d}}\left(S_{\lambda_{1}}(s) S_{\lambda_{2}}(t) f+L\left(\lambda_{2}\right) t-L\left(\lambda_{1}\right) s\right) \\
& \leq \inf _{\lambda_{2} \in \mathbb{R}^{d}} \sup _{\lambda_{1} \in \mathbb{R}^{d}}\left(S_{\lambda_{2}}(t)\left(S_{\lambda_{1}}(s) f-L\left(\lambda_{1}\right) s\right)+L\left(\lambda_{2}\right) t\right) \\
& \leq \inf _{\lambda_{2} \in \mathbb{R}^{d}}\left(S_{\lambda_{2}}(t) I(s) f+L\left(\lambda_{2}\right) t\right)=I^{-}(t) I(s) f .
\end{aligned}
$$

3. For every $s, t \geq 0$ and $f \in C_{0}$,

$$
\begin{aligned}
I(s+t) f & =\sup _{\lambda \in \mathbb{R}^{d}}\left(S_{\lambda}(s+t) f-L(\lambda)(s+t)\right) \\
& =\sup _{\lambda \in \mathbb{R}^{d}}\left(S_{\lambda}(s) S_{\lambda}(t) f-L(\lambda) s-L(\lambda) t\right) \\
& =\sup _{\lambda \in \mathbb{R}^{d}}\left(S_{\lambda}(s)\left(S_{\lambda}(t) f-L(\lambda) t\right)-L(\lambda) s\right) \\
& \leq \sup _{\lambda \in \mathbb{R}^{d}}\left(S_{\lambda}(s) I(t) f-L(\lambda) s\right)=I(s) I(t) f .
\end{aligned}
$$

Second, we show $S(t) x=\sup _{\pi \in P_{t}} I(\pi) x$ for all $(x, t) \in X \times \mathbb{R}_{+}$. To do so, we use Lemma 15. Clearly, $I(t)$ is continuous from below for all $t \geq 0$. Next, we show that the mapping $I(\cdot) f$ is continuous for all $f \in C_{0}$ : For every $f \in C_{0}^{2}$, it follows from Lemma 6.1 and Itô's formula that there exists $r \geq 0$ with

$$
\begin{aligned}
\|I(t) f-I(s) f\|_{\infty} & \leq \sup _{\lambda \in B(0, r) \cap\{L<\infty\}}\left(\left\|S_{\lambda}(t-s) f\right\|_{\infty}+L(\lambda)(t-s)\right) \\
& \leq \sup _{\lambda \in B(0, r) \cap\{L<\infty\}}\left(|\lambda| \cdot\|\nabla f\|_{\infty}+\frac{1}{2}\|\Delta f\|_{\infty}+L(\lambda)\right)(t-s)
\end{aligned}
$$

for all $0 \leq s \leq t$. Moreover, the assumption on $L$ implies

$$
\sup _{\lambda \in B(0, r) \cap\{L<\infty\}}\left(|\lambda| \cdot\|\nabla f\|_{\infty}+\frac{1}{2}\|\Delta f\|_{\infty}+L(\lambda)\right)<\infty .
$$

Since $C_{0}^{2} \subset C_{0}$ is dense and $(I(t))_{t \geq 0}$ is a family of contractions, the mapping $I(\cdot) f$ is also continuous for arbitrary $f \in C_{0}$, see the proof of Lemma 2.10 . It remains to show that, for every $t \geq 0$, the operator

$$
T(t): C_{0} \rightarrow C_{0}, f \mapsto \sup _{\pi \in P_{t}} I(\pi) f
$$

is well-defined. Lemma 3.2 implies $\left\{I(\pi): \pi \in P_{t}\right\} \subset \operatorname{Lip}_{0}(c)$, and therefore $T(t) f \in$ $\operatorname{Lip}_{0}(c)$ for all $c, t \geq 0$ and $f \in \operatorname{Lip}_{0}(c)$. Since $T(t): C_{0} \rightarrow \mathcal{L}^{\infty}$ is continuous and $\operatorname{Lip}_{0} \subset C_{0}$ is dense, we obtain $T(t): C_{0} \rightarrow C_{0}$ for all $t \geq 0$. We can apply Lemma 15 and conclude that $S(t) x=T(t) x$ for all $(x, t) \in X \times \mathbb{R}_{+}$. Hence, Assumption 5.2 is satisfied and Theorem 5.3 yields $\mathcal{L}^{S}=\mathcal{L}^{I}$ and $S(t): \mathcal{L}_{\text {sym }}^{S} \rightarrow \mathcal{L}_{\text {sym }}^{S}$ for all $t \geq 0$.

Third, we show $\mathcal{L}_{\text {sym }}^{I} \subset\left\{f \in W^{1, \infty} \cap C_{0}: \Delta f\right.$ exists in $\left.L^{\infty}\right\}$. Let $f \in \mathcal{L}_{\text {sym }}^{I}$. By definition, there exist $t_{0}>0$ and $c \geq 0$ such that

$$
\|I(t) f-f\|_{\infty} \leq c t \quad \text { and } \quad\|I(t)(-f)+f\|_{\infty} \leq c t \quad \text { for all } t \in\left[0, t_{0}\right] .
$$


For every $\lambda \in \mathbb{R}^{d}$ and $t \in\left[0, t_{0}\right]$,

$$
\begin{aligned}
-(c+L(\lambda)) t & \leq-(I(t)(-f)+f+L(\lambda) t) \leq-\left(S_{\lambda}(t)(-f)+f\right) \\
& =S_{\lambda}(t) f-f \leq I(t) f-f+L(\lambda) t \leq(c+L(\lambda)) t,
\end{aligned}
$$

and therefore $\left\|S_{\lambda}(t) f-f\right\|_{\infty} \leq(c+L(\lambda)) t$. Let $\eta \in C_{c}^{\infty}$ with $\eta \geq 0, \operatorname{supp}(\eta) \subset B(0,1)$ and $\int_{\mathbb{R}^{d}} \eta(x) \mathrm{d} x=1$. For every $n \in \mathbb{N}$ and $x \in \mathbb{R}^{d}$, we define $\eta_{n}(x):=n^{d} \eta(n x)$ and

$$
f_{n}(x):=\left(f * \eta_{n}\right)(x)=\int_{\mathbb{R}^{d}} f(x-y) \eta_{n}(y) \mathrm{d} y .
$$

Let $\lambda \in \mathbb{R}^{d}$ and $t \geq 0$. Fubini's theorem implies

$$
\begin{aligned}
\left|S_{\lambda}(t) f_{n}-f_{n}\right|(x) & =\left|\mathbb{E}\left[\int_{\mathbb{R}^{d}} f\left(x+W_{t}+\lambda t-y\right) \eta_{n}(y) d y\right]-f_{n}(x)\right| \\
& =\left|\int_{\mathbb{R}^{d}} \mathbb{E}\left[f\left(x+W_{t}+\lambda t-y\right)\right] \eta_{n}(y) d y-f_{n}(x)\right| \\
& =\left|\left(S_{\lambda}(t) f-f\right) * \eta_{n}\right|(x) \leq\left\|S_{\lambda}(t) f-f\right\|_{\infty} \leq(c+L(\lambda)) t .
\end{aligned}
$$

Since $f_{n} \in C_{0}^{2} \subset D\left(A_{\lambda}\right)$, we obtain

$$
\left\|A_{\lambda} f_{n}\right\|_{\infty} \leq c+L(\lambda) \text { for all } n \in \mathbb{N} .
$$

Moreover, for every $n \in \mathbb{N}$, we have the identities

$$
\begin{aligned}
\Delta f_{n} & =\frac{1}{2} \Delta f_{n}+\nabla_{\lambda} f_{n}+\frac{1}{2} \Delta f_{n}+\nabla_{-\lambda} f_{n}=A_{\lambda} f_{n}+A_{-\lambda} f_{n}, \\
2 \nabla_{\lambda} f_{n} & =\frac{1}{2} \Delta f_{n}+\nabla_{\lambda} f_{n}+\frac{1}{2} \Delta\left(-f_{n}\right)+\nabla_{-\lambda}\left(-f_{n}\right)=A_{\lambda} f_{n}+A_{-\lambda}\left(-f_{n}\right) .
\end{aligned}
$$

It follows from inequality (6.2) and inequality (6.3) that $\sup _{n \in \mathbb{N}}\left\|\Delta f_{n}\right\|_{\infty}<\infty$, since the assumptions on $L$ ensure the existence of $\lambda \in \mathbb{R}^{d}$ with $L( \pm \lambda)<\infty$. Furthermore, we use inequality (6.2), inequality (6.4) and the assumption on $L$ to estimate

$$
\begin{aligned}
\left\|\nabla f_{n}\right\|_{\infty} & =\frac{1}{\varepsilon} \sup _{\{|\lambda|=\varepsilon\}}\left\|\nabla_{\lambda} f_{n}\right\|_{\infty} \leq \frac{1}{2 \varepsilon} \sup _{\{|\lambda|=\varepsilon\}}\left(\left\|A_{\lambda} f_{n}\right\|_{\infty}+\left\|A_{\lambda}\left(-f_{n}\right)\right\|_{\infty}\right) \\
& \leq \frac{1}{\varepsilon} \sup _{\{|\lambda|=\varepsilon\}}(c+L(\lambda))<\infty \quad \text { for all } n \in \mathbb{N} .
\end{aligned}
$$

By Banach-Alaoglu's theorem, there exist functions $g, g_{i} \in L^{\infty}$ such that $\Delta f_{n_{k}} \rightarrow g$ and $\partial_{i} f_{n} \rightarrow g_{i}$ as $k \rightarrow \infty$ in the weak*-topology for a suitable subsequence. This implies $f \in\left\{h \in W^{1, \infty} \cap C_{0}: \Delta h\right.$ exists in $\left.L^{\infty}\right\}$ with $\Delta f=g$ and $\partial_{i} f=g_{i}$ for $i=1, \ldots, d$.

Forth, we show $\left\{f \in W^{1, \infty} \cap C_{0}: \Delta f\right.$ exists in $\left.L^{\infty}\right\} \subset \mathcal{L}_{\text {sym }}^{I}$. Let $f \in W^{1, \infty} \cap C_{0}$ such that $\Delta f$ exists in $L^{\infty}$. By Lemma 6.1, there exists $r \geq 0$ such that

$$
I(t) f=\sup _{\lambda \in B(0, r)} I_{\lambda}(t) f \quad \text { for all } t \geq 0 .
$$

Let $t \geq 0, \lambda \in B(0, r)$ and $f_{n}:=f * \eta_{n}$ for all $n \in \mathbb{N}$. We use Itô's formula, $\nabla f_{n}=$ $(\nabla f) * \eta_{n}$ and $\Delta f_{n}=(\Delta f) * \eta_{n}$ to estimate

$$
\begin{aligned}
I_{\lambda}(t) f-f=\lim _{n \rightarrow \infty}\left(I_{\lambda}(t) f_{n}-f_{n}\right) & \leq \sup _{n \in \mathbb{N}}\left(|\lambda| \cdot\left\|\nabla f_{n}\right\|_{\infty}+\frac{1}{2}\left\|\Delta f_{n}\right\|_{\infty}-L(\lambda)\right) t \\
& \leq\left(r \cdot\|\nabla f\|_{\infty}+\frac{1}{2}\|\Delta f\|_{\infty}\right) t .
\end{aligned}
$$


Taking the supremum over $\lambda \in B(0, r)$ yields

$$
I(t) f-f \leq\left(r \cdot\|\nabla f\|_{\infty}+\frac{1}{2}\|\Delta f\|_{\infty}\right) t \quad \text { for all } t \geq 0 .
$$

For the lower bound, we choose $\lambda \in \mathbb{R}^{d}$ with $L(\lambda)=0$ and obtain

$$
I(t) f-f \geq S_{\lambda}(t) f-f \geq-\left(\left(|\lambda| \cdot\|\nabla f\|_{\infty}+\frac{1}{2}\|\Delta f\|_{\infty}\right) t \quad \text { for all } t \geq 0 .\right.
$$

This shows $f \in \mathcal{L}^{I}$. Applying the same argument on $-f$ yields $f \in \mathcal{L}_{\text {sym }}^{I}$.

Remark 6.4 1. For functions $f \in \mathcal{L}_{\text {sym }}^{S}$ the Laplacian and gradient are defined in the distributional sense and $\frac{1}{2} \Delta f+H(\nabla f) \in L^{\infty}$. By extending the semigroup $(S(t))_{t \geq 0}$ from $C_{0}$ to an exponential Orlicz heart, one can show that the Cauchy problem

$$
\partial_{t} u(t)=\frac{1}{2} \Delta u(t)+H(\nabla u(t)) \quad \text { for all } t \geq 0, \quad u(0)=f,
$$

has a unique classical solution, which is represented by the extended semigroup. Here, the initial value $f \in \mathcal{L}_{\text {sym }}^{S}$ is chosen such that the generator $A f$ is defined w.r.t. to the Orlicz norm, which is weaker than the supremum norm. For details, we refer to [3]. A sublinear version of this example was studied in [14].

2. The explicit description of the symmetric Lipschitz set in the previous theorem relies only on elementary estimates and Banach-Alaoglu's theorem. Nonetheless, by using the results in [23], we obtain

$$
\mathcal{L}_{\text {sym }}^{S}=\left\{f \in \bigcap_{p \geq 1} W_{\text {loc }}^{2, p} \cap C_{0}: \Delta f \in L^{\infty}\right\},
$$

i.e., the symmetric Lipschitz set equals the domain of the Laplacian in $C_{0}$.

Proof Let $\left\{f \in \bigcap_{p \geq 1} W_{\text {loc }}^{2, p} \cap C_{0}: \Delta f \in L^{\infty}\right\}$. By [23, Theorem 3.1.7], it holds $f \in W^{1, \infty}$, and therefore $f \in \mathcal{L}_{\text {sym }}^{S}$. Now, let $f \in \mathcal{L}_{\text {sym }}^{I}$ and $f_{n}:=f * \eta_{n}$ for all $n \in \mathbb{N}$. Fix $p>d$. By [23, Theorem 3.1.6], there exist $c \geq 0$ and $\varepsilon>0$ such that, for all $n \in \mathbb{N}$,

$$
\sup _{x \in \mathbb{R}^{d}}\left\|D^{2} f_{n}\right\|_{L^{p}(B(x, \varepsilon))} \leq c\left(\left\|f_{n}\right\|_{\infty}+\left\|\Delta f_{n}\right\|_{\infty}\right) \leq c\left(\|f\|_{\infty}+\|\Delta f\|_{\infty} .\right.
$$

This implies $\sup _{n \in \mathbb{N}}\left\|f_{n}\right\|_{W^{2, p}(B(0, r))}<\infty$ for all $r \geq 0$ and $n \in \mathbb{N}$. Taking the limit $n \rightarrow \infty$ yields $f \in W^{2, p}(B(0, r))$ for all $r \geq 0$, i.e., $f \in W_{\text {loc }}^{2, p}$. Since $W_{\text {loc }}^{2, q} \subset$ $W_{\text {loc }}^{2, p}$ for all $p \leq q$, we obtain $f \in \bigcap_{p \geq 1} W_{\text {loc }}^{2, p}$. The last part of the claim follows from [23, Theorem 3.1.7].

\subsection{Geometric Brownian Motion}

In this subsection, we construct a semigroup, which corresponds to a geometric Brownian motion with uncertain drift and volatility. Based on the Nisio approach, this example has been studied in [24], where the authors obtain a viscosity solution for the associated Cauchy problem. In contrast to the previous example, we have to weaken the supremum norm by adding a weight function.

Let $\left(W_{t}\right)_{t \geq 0}$ be a 1 -dimensional Brownian motion on a probability space $(\Omega, \mathcal{F}, \mathbb{P})$ and $p>1$. We choose the weight function

$$
\kappa: \mathbb{R} \rightarrow(0, \infty), x \mapsto\left(1+|x|^{p}\right)^{-1} .
$$


Let $\Lambda \subset \mathbb{R} \times \mathbb{R}_{+}$be a bounded set. For every $f \in \mathrm{UC}_{\kappa}\left(\mathbb{R}^{d} ; \mathbb{R}\right), t \geq 0$ and $x \in \mathbb{R}$, we define

$$
(I(t) f)(x):=\sup _{\lambda \in \Lambda} \mathbb{E}\left[f\left(X_{t}^{\lambda, x}\right)\right],
$$

where $X_{t}^{\lambda, x}:=x X_{t}^{\lambda}$ and $X_{t}^{\lambda}:=\exp \left(\left(\mu-\frac{\sigma^{2}}{2}\right) t+\sigma W_{t}\right)$ for $\lambda:=\left(\mu, \sigma^{2}\right)$. Furthermore, for every $\lambda \in \Lambda$, let $\left(S_{\lambda}(t)\right)_{t \geq 0}$ be the linear semigroup given by

$$
\left(S_{\lambda}(t) f\right)(x):=\mathbb{E}\left[f\left(X_{t}^{\lambda, x}\right)\right] \text { for all } t \geq 0, f \in \mathrm{UC}_{\kappa} \text { and } x \in \mathbb{R} .
$$

We start with two auxiliary lemmas.

Lemma 6.5 For every $\lambda:=\left(\mu, \sigma^{2}\right) \in \Lambda, t \geq 0, x \in \mathbb{R}$ and $f \in \mathrm{UC}_{\kappa}$,

$$
\mathbb{E}\left[\left|X_{t}^{\lambda}\right|\right]=e^{\mu t}, \quad \mathbb{E}\left[\left|X_{t}^{\lambda}\right|^{p}\right] \leq e^{\omega t} \quad \text { and } \quad \mathbb{E}\left[\left|f\left(X_{t}^{\lambda, x}\right)\right|\right] \leq\|f\|_{\kappa}\left(1+|x|^{p}\right) e^{\omega t},
$$

where $\omega:=\sup _{\lambda \in \Lambda} p\left(\mu+\frac{(p-1) \sigma^{2}}{2}\right)^{+}<\infty$.

Proof Fix $t \geq 0$ and $\lambda \in \Lambda$. We use the moment generating function of the normal distribution to compute

$$
\mathbb{E}\left[\left|X_{t}^{\lambda}\right|\right]=\exp \left(\left(\mu-\frac{\sigma^{2}}{2}\right) t\right) \mathbb{E}\left[\exp \left(\sigma W_{t}\right)\right]=\exp \left(\left(\mu-\frac{\sigma^{2}}{2}\right) t\right) \exp \left(\frac{\sigma^{2} t}{2}\right)=e^{\mu t},
$$

and

$$
\mathbb{E}\left[\left|X_{t}^{\lambda}\right|^{p}\right]=\exp \left(p\left(\mu-\frac{\sigma^{2}}{2}\right) t\right) \mathbb{E}\left[\exp \left(p \sigma W_{t}\right)\right]=\exp \left(p\left(\mu+\frac{(p-1) \sigma^{2}}{2}\right) t\right) \leq e^{\omega t} .
$$

Let $f \in \mathrm{UC}_{\kappa}$ and $x \in \mathbb{R}^{d}$. Since $\left|f\left(X_{t}^{\lambda, x}\right)\right| \kappa\left(X_{t}^{\lambda, x}\right) \leq\|f \kappa\|_{\infty}=\|f\|_{\kappa}$, we obtain $\left|f\left(X_{t}^{\lambda, x}\right)\right| \leq\|f\|_{\kappa}\left(1+\left|X_{t}^{\lambda, x}\right|^{p}\right)$. We use the previous estimate to conclude

$$
\mathbb{E}\left[\left|f\left(X_{t}^{\lambda, x}\right)\right|\right] \leq\|f\|_{\kappa}\left(1+|x|^{p} \mathbb{E}\left[\left|X_{t}^{\lambda}\right|^{p}\right]\right) \leq\|f\|_{\kappa}\left(1+|x|^{p}\right) e^{\omega t} .
$$

Lemma 6.5 ensures that $S_{\lambda}(t) f: \mathbb{R} \rightarrow \mathbb{R}$ and $I(t) f: \mathbb{R} \rightarrow \mathbb{R}$ are well-defined functions for all $\lambda \in \Lambda, t \geq 0$ and $f \in \mathrm{UC}_{\kappa}$.

Lemma 6.6 For every $q \in[1, \infty)$ and $\varepsilon>0$,

$$
\lim _{t \downarrow 0} \sup _{\lambda \in \Lambda} \mathbb{P}\left(\left|\left(X_{t}^{\lambda}\right)^{q}-1\right| \geq \varepsilon\right)=0 \quad \text { and } \quad \lim _{t \downarrow 0} \sup _{\lambda \in \Lambda} \mathbb{E}\left[\left|\left(X_{t}^{\lambda}\right)^{q}-1\right|\right]=0 \text {. }
$$

Proof Let $q \in[1, \infty)$ and $\varepsilon>0$. Choose $\delta>0$ such that $\left|e^{x}-1\right|<\varepsilon$ for all $x \in(-\delta, \delta)$. Then, for every $t \geq 0$ and $\lambda \in \Lambda$,

$$
\mathbb{P}\left(\left|\left(X_{t}^{\lambda}\right)^{q}-1\right| \geq \varepsilon\right) \leq \mathbb{P}\left(q\left|\left(\mu-\frac{\sigma^{2}}{2}\right) t+\sigma W_{t}\right| \geq \delta\right) .
$$

Let $c:=\sup _{\lambda \in \Lambda} \max \left\{q \sigma, q\left|\mu-\sigma^{2} / 2\right|\right\}$. Then, for every $t \in[0, \delta / 2 c]$ and $\lambda \in \Lambda$,

$$
q\left|\left(\mu-\frac{\sigma^{2}}{2}\right) t+\sigma W_{t}\right| \geq \delta \text { implies } \quad\left|W_{t}\right| \geq \frac{\delta}{2 c}>0 .
$$

We conclude

$$
\sup _{\lambda \in \Lambda} \mathbb{P}\left(\left|\left(X_{t}^{\lambda}\right)^{q}-1\right| \geq \varepsilon\right) \leq \mathbb{P}\left(\left|W_{t}\right| \geq \frac{\delta}{2 c}\right) \quad \text { for all } t \in\left[0, \frac{\delta}{2 c}\right] .
$$

Since the right hand side of the previous inequality converges to zero as $t \downarrow 0$, we obtain the first part of the claim. 
Furthermore, by Lemma 6.5, the set $\left\{\left|\left(X_{t}^{\lambda}\right)^{q}-1\right|: t \in[0,1], \lambda \in \Lambda\right\}$ is bounded in $L^{2}(\mathbb{P})$, and therefore uniformly integrable. Hence, the second part of the claim follows from the first one, similar to the Vitali convergence theorem.

Let $C_{c}^{2}$ be the set of all twice continuously differentiable functions $f: \mathbb{R} \rightarrow \mathbb{R}$ with compact support.

Theorem 6.7 The family $(I(t))_{t \geq 0}$ satisfies Assumptions 2.1 and 2.4, i.e., Theorem 2.5 yields a semigroup $(S(t))_{t \geq 0}$ on $\mathrm{UC}_{\kappa}$ associated to $(I(t))_{t \geq 0}$. Furthermore, it holds $C_{c}^{2} \subset$ $D(A)$ with

$$
(A f)(x)=\sup _{\lambda \in \Lambda}\left(\frac{1}{2} \sigma^{2} x^{2} f^{\prime \prime}(x)+\mu x f^{\prime}(x)\right) \quad \text { for all } f \in C_{c}^{2} \text { and } x \in \mathbb{R} .
$$

Proof First, we show $I(t): \operatorname{Lip}_{b}(c) \rightarrow \operatorname{Lip}_{b}\left(e^{\omega t} c\right)$ for all $c, t \geq 0$. Let $c, t \geq 0$ and $f \in \operatorname{Lip}_{b}(c)$. Lemma 6.5 implies

$\left|\left(S_{\lambda}(t) f\right)(x)-\left(S_{\lambda}(t) f\right)(y)\right| \leq \mathbb{E}\left[\left|f\left(x X_{t}^{\lambda}\right)-f\left(y X_{t}^{\lambda}\right)\right|\right] \leq c|x-y| \mathbb{E}\left[\left|X_{t}^{\lambda}\right|\right] \leq c e^{\omega t}|x-y|$ for all $\lambda \in \Lambda$ and $x, y \in \mathbb{R}$. Furthermore, it holds $\|I(t) f\|_{\infty} \leq\|f\|_{\infty} \leq e^{\omega t} c$.

Second, we verify Assumption 2.1.

1. Clearly, $I(0)=\operatorname{id}_{U_{\kappa}}$.

2. Let $f \in \mathrm{UC}_{\kappa}, t \geq 0, \lambda \in \Lambda$ and $x \in \mathbb{R}$. It follows from Lemma 6.5 that

$$
\left|S_{\lambda}(t) f(x)\right| \kappa(x) \leq\|f\|_{\kappa}\left(1+|x|^{p}\right) e^{\omega t}\left(1+|x|^{p}\right)^{-1}=e^{\omega t}\|f\|_{\kappa} .
$$

Hence, we obtain $\left\|S_{\lambda}(t) f\right\|_{\kappa} \leq e^{\omega t}\|f\|_{\kappa}$, and therefore

$$
\|I(t) f\|_{\kappa} \leq \sup _{\lambda \in \Lambda}\left\|S_{\lambda}(t) f\right\|_{\kappa} \leq e^{\omega t}\|f\|_{\kappa} .
$$

3. For every $f, g \in \mathrm{UC}_{\kappa}$ and $t \geq 0$,

$$
\|I(t) f-I(t) g\|_{\kappa} \leq \sup _{\lambda \in \Lambda}\left\|S_{\lambda}(t)(f-g)\right\|_{\kappa} \leq e^{\omega t}\|f-g\|_{\kappa} .
$$

In particular, we obtain $I(t): \mathrm{UC}_{\kappa} \rightarrow \mathrm{UC}_{\kappa}$, because $I(t): \operatorname{Lip}_{b} \rightarrow \operatorname{Lip}_{b}, \operatorname{Lip}_{b} \subset \mathrm{UC}_{\kappa}$ is dense and $I(t)$ is Lipschitz continuous.

Third, we verify Assumption 2.4. Let $f \in C_{c}^{2}$ and choose $r \geq 0$ with $\operatorname{supp}(f) \subset[-r, r]$. Fix $\lambda \in \Lambda, t \geq 0$ and $x \in \mathbb{R}$. Itô's formula implies

$$
\left(S_{\lambda}(t) f\right)(x)-f(x)=\mu \mathbb{E}\left[\int_{0}^{t} X_{s}^{\lambda, x} f^{\prime}\left(X_{s}^{\lambda, x}\right) d s\right]+\frac{\sigma^{2}}{2} \mathbb{E}\left[\int_{0}^{t}\left(X_{s}^{\lambda, x}\right)^{2} f^{\prime \prime}\left(X_{s}^{\lambda, x}\right) d s\right] .
$$

Hence, we can estimate

$$
\|I(t) f-f\|_{\kappa} \leq\|I(t) f-f\|_{\infty} \leq \sup _{\lambda \in \Lambda}\left\|S_{\lambda}(t) f-f\right\|_{\infty} \leq c t,
$$

where $c:=\sup _{\lambda \in \Lambda}\left(\mu r\left\|f^{\prime}\right\|_{\infty}+\frac{\sigma^{2}}{2} r^{2}\left\|f^{\prime \prime}\right\|_{\infty}\right)$. Since $I(t): \operatorname{Lip}_{b}(c) \rightarrow \operatorname{Lip}_{b}\left(e^{\omega t} c\right)$ for all $c, t \geq 0$, it follows from Lemma 3.3 that the sequence $\left(I\left(\pi_{n}^{t}\right) f\right)_{n \in \mathbb{N}}$ is relatively compact in $\mathrm{UC}_{\kappa}$ for all $f \in \operatorname{Lip}_{b}$ and $t \in \mathcal{T}$. Furthermore, by Lemma 3.4, we can choose a countable set $\mathcal{D} \subset C_{c}^{2}$, which is dense in $\mathrm{UC}_{\kappa}$. By Theorem 2.5 there exists a semigroup $(S(t))_{t \geq 0}$ on $\mathrm{UC}_{\kappa}$ associated to $(I(t))_{t \geq 0}$. 
Forth, for every $f \in C_{c}^{2}$, we show that

$$
\lim _{t \downarrow 0}\left\|\frac{I(t) f-f}{t}-g\right\|_{\kappa}=0,
$$

where $g:=\sup _{\lambda \in \Lambda} g_{\lambda}$ and $g_{\lambda}(x):=\left(\frac{\sigma^{2}}{2} x^{2} f^{\prime \prime}(x)+\mu x f^{\prime}(x)\right)$ for all $\lambda \in \Lambda$ and $x \in \mathbb{R}$. It holds

$$
\left\|\frac{I(t) f-f}{t}-g\right\|_{\kappa} \leq \sup _{\lambda \in \Lambda}\left\|\frac{S_{\lambda}(t) f-f}{t}-g_{\lambda}\right\|_{\kappa} \quad \text { for all } t>0,
$$

and Itô's formula implies

$$
\begin{aligned}
\left|\frac{S_{\lambda}(t) f-f}{t}-g_{\lambda} f\right|(x) \leq & \frac{|\mu|}{t} \int_{0}^{t} \mathbb{E}\left[\left|X_{s}^{\lambda, x} f^{\prime}\left(X_{s}^{\lambda, x}\right)-x f^{\prime}(x)\right|\right] d s \\
& +\frac{\sigma^{2}}{2 t} \int_{0}^{t} \mathbb{E}\left[\left|\left(X_{s}^{\lambda, x}\right)^{2} f^{\prime \prime}\left(X_{s}^{\lambda, x}\right)-x^{2} f^{\prime \prime}(x)\right|\right] d s
\end{aligned}
$$

for all $\lambda \in \Lambda, t>0$ and $x \in \mathbb{R}$. To estimate the first term on the right hand side, we show

$$
\lim _{t \downarrow 0} \sup _{\lambda \in \Lambda} \sup _{x \in \mathbb{R}} \frac{|\mu|}{t} \int_{0}^{t} \mathbb{E}\left[\left|X_{s}^{\lambda, x} f^{\prime}\left(X_{s}^{\lambda, x}\right)-x f^{\prime}(x)\right|\right] \mathrm{d} s=0 .
$$

Let $\varepsilon>0, c:=1+\sup _{\lambda \in \Lambda}|\mu|$, and choose $r \geq 0$ with $\operatorname{supp}(f) \subset[-r, r]$. Moreover, since $f^{\prime}$ is uniformly continuous, there exists $\delta>0$ such that

$$
\left|f^{\prime}(x)-f^{\prime}(y)\right|<\frac{\varepsilon}{6 c r} \text { for all } x, y \in \mathbb{R} \text { with }|x-y|<2 \delta r .
$$

Fix $\lambda \in \Lambda$ and $x \in \mathbb{R}$. We distinguish two cases:

1. Let $|x| \leq 2 r$. Then, for every $s \geq 0$,

$$
\begin{aligned}
& \mathbb{E}\left[\left|X_{s}^{\lambda, x} f^{\prime}\left(X_{s}^{\lambda, x}\right)-x f^{\prime}(x)\right|\right]=|x| \mathbb{E}\left[\left|X_{s}^{\lambda} f^{\prime}\left(X_{s}^{\lambda, x}\right)-f^{\prime}(x)\right|\right] \\
& \leq 2 r \mathbb{E}\left[\left|X_{s}^{\lambda}-1\right| \cdot\left|f^{\prime}\left(X_{s}^{\lambda, x}\right)\right|\right]+2 r \mathbb{E}\left[\left|f^{\prime}\left(X_{s}^{\lambda, x}\right)-f^{\prime}(x)\right| \mathbb{1}_{\left\{\left|X_{s}^{\lambda}-1\right| \geq \delta\right\}}\right] \\
& \quad+2 r \mathbb{E}\left[\left|f^{\prime}\left(X_{s}^{\lambda, x}\right)-f^{\prime}(x)\right| \mathbb{1}_{\left\{\left|X_{s}^{\lambda}-1\right|<\delta\right\}}\right] \\
& \leq 2 r\left\|f^{\prime}\right\|_{\infty} \mathbb{E}\left[\left|X_{s}^{\lambda}-1\right|\right]+4 r\left\|f^{\prime}\right\|_{\infty} \mathbb{P}\left(\left|X_{s}^{\lambda}-1\right| \geq \delta\right) \\
& \quad+2 r \mathbb{E}\left[\left|f^{\prime}\left(X_{s}^{\lambda, x}\right)-f^{\prime}(x)\right| \mathbb{1}_{\left\{\left|X_{s}^{\lambda}-1\right|<\delta\right\}}\right] .
\end{aligned}
$$

By Lemma 6.6, there exists $s_{0}>0$, independent of $\lambda$ and $x$, such that

$$
2 r\left\|f^{\prime}\right\|_{\infty} \mathbb{E}\left[\left|X_{s}^{\lambda}-1\right|\right] \leq \frac{\varepsilon}{3 c} \quad \text { and } \quad 4 r\left\|f^{\prime}\right\|_{\infty} \mathbb{P}\left(\left|X_{s}^{\lambda}-1\right| \geq \delta\right) \leq \frac{\varepsilon}{3 c}
$$

for all $s \in\left[0, s_{0}\right]$. On the set $\left\{\left|X_{s}^{\lambda}-1\right|<\delta\right\}$, it holds $\left|X_{s}^{\lambda, x}-x\right|=|x| \cdot\left|X_{s}^{\lambda}-1\right|<2 r \delta$, and therefore $\left|f^{\prime}\left(X_{s}^{\lambda, x}\right)-f^{\prime}(x)\right|<\frac{\varepsilon}{6 c r}$. This implies

$$
2 r \mathbb{E}\left[\left|f^{\prime}\left(X_{s}^{\lambda, x}\right)-f^{\prime}(x)\right| \mathbb{1}_{\left\{\left|X_{s}^{\lambda}-1\right|<\delta\right\}}\right] \leq \frac{\varepsilon}{3 c} .
$$

We combine inequality (6.7) and inequality (6.8) to conclude

$$
\sup _{s \in\left[0, s_{0}\right]} \sup _{\lambda \in \Lambda} \sup _{x \in[-2 r, 2 r]} \mathbb{E}\left[\left|X_{s}^{\lambda, x} f^{\prime}\left(X_{s}^{\lambda, x}\right)-x f^{\prime}(x)\right|\right] \leq \frac{\varepsilon}{c} .
$$

2. Let $|x|>2 r$. Then, for every $s \geq 0$,

$$
\begin{aligned}
\mathbb{E}\left[\left|X_{s}^{\lambda, x} f^{\prime}\left(X_{s}^{\lambda, x}\right)-x f^{\prime}(x)\right|\right] & =\mathbb{E}\left[\left|X_{s}^{\lambda, x} f^{\prime}\left(X_{s}^{\lambda, x}\right)\right| \mathbb{1}_{\left\{\left|X_{s}^{\lambda, x}\right| \leq r\right\}}\right] \\
& \leq r\left\|f^{\prime}\right\|_{\infty} \mathbb{P}\left(\left|X_{s}^{\lambda, x}\right| \leq r\right),
\end{aligned}
$$


because $\operatorname{supp}(f) \subset[-r, r]$. Furthermore, we use $|x|>2 r$ to estimate

$$
\mathbb{P}\left(\left|X_{s}^{\lambda, x}\right| \leq r\right)=\mathbb{P}\left(|x| \cdot\left|X_{s}^{\lambda}\right| \leq r\right) \leq \mathbb{P}\left(\left|X^{\lambda}\right|<\frac{1}{2}\right) .
$$

By Lemma 6.6, there exists $s_{1} \in\left(0, s_{0}\right]$, independent of $\lambda$ and $x$, such that

$$
r\left\|f^{\prime}\right\|_{\infty} \mathbb{P}\left(\left|X_{s}^{\lambda}\right|<\frac{1}{2}\right) \leq \frac{\varepsilon}{c} \quad \text { for all } s \in\left[0, s_{1}\right] .
$$

We obtain

$$
\sup _{s \in\left[0, s_{1}\right] \lambda \in \Lambda} \sup _{\lambda \in[-2 r, 2 r]^{c}} \sup _{x \in[} \mathbb{E}\left[\left|X_{s}^{\lambda, x} f^{\prime}\left(X_{s}^{\lambda, x}\right)-x f^{\prime}(x)\right|\right] \leq \frac{\varepsilon}{c} .
$$

Combining inequality (6.9) and inequality (6.10) with the definition of $c$ yields

$$
\sup _{\lambda \in \Lambda} \sup _{x \in \mathbb{R}} \frac{|\mu|}{t} \int_{0}^{t} \mathbb{E}\left[\left|X_{s}^{\lambda, x} f^{\prime}\left(X_{s}^{\lambda, x}\right)-x f^{\prime}(x)\right|\right] \mathrm{d} s \leq \varepsilon \quad \text { for all } t \in\left[0, s_{1}\right] .
$$

By similar arguments, it follows from Lemma 6.6 with $q=2$ that

$$
\lim _{t \downarrow 0} \sup _{\lambda \in \Lambda} \sup _{x \in \mathbb{R}} \frac{\sigma^{2}}{2 t} \int_{0}^{t} \mathbb{E}\left[\left|\left(X_{s}^{\lambda, x}\right)^{2} f^{\prime \prime}\left(X_{s}^{\lambda, x}\right)-x^{2} f^{\prime \prime}(x)\right|\right] d s=0 .
$$

As seen before, Eq. 6.5 is a consequence of inequality (6.6) and inequality (6.11).

Fifth, it follows from Theorem 2.5 that Assumption 4.1 is satisfied. In addition, by Lemma 4.4, condition (4.2) holds for all $f, g \in \mathrm{UC}_{\kappa}$. Hence, Theorem 4.3 implies $C_{c}^{2} \subset D(A)$ with

$$
(A f)(x)=\sup _{\lambda \in \Lambda}\left(\frac{1}{2} \sigma^{2} x^{2} f^{\prime \prime}(x)+\mu x f^{\prime}(x)\right) \quad \text { for all } f \in C_{c}^{2} \text { and } x \in \mathbb{R} .
$$

\subsection{G-expectation}

In this subsection, we construct a semigroup corresponding to a Brownian motion with uncertain drift and volatility, which is the so-called $G$-Brownian motion, see [29, 30]. Let $\left(W_{t}\right)_{t \geq 0}$ be a $d$-dimensional Brownian motion on a probability space $(\Omega, \mathcal{F}, \mathbb{P})$. We choose the weight function

$$
\kappa: \mathbb{R}^{d} \rightarrow(0, \infty), x \mapsto\left(1+|x|^{2}\right)^{-1} .
$$

Let $\Lambda \subset \mathbb{R}^{d} \times \mathbb{S}_{+}^{d}$ be a bounded set, where $\mathbb{S}_{+}^{d}$ denotes the set of all symmetric, positive semi-definite $d \times d$-matrices. For every $t \geq 0, f \in \mathrm{UC}_{\kappa}\left(\mathbb{R}^{d} ; \mathbb{R}\right)$ and $x \in \mathbb{R}^{d}$, we define

$$
(I(t) f)(x):=\sup _{\lambda \in \Lambda} \mathbb{E}\left[f\left(x+\sigma W_{t}+\mu t\right)\right],
$$

where $\lambda:=\left(\mu, \sigma^{2}\right)$ and $\sigma:=\sqrt{\sigma^{2}}$ denotes the matrix square root of $\sigma^{2}$. Moreover, for every $\lambda \in \Lambda$, let $\left(S_{\lambda}(t)\right)_{t \geq 0}$ be the linear semigroup defined by

$$
\left(S_{\lambda}(t) f\right)(x):=\mathbb{E}\left[f\left(x+\sigma W_{t}+\mu t\right)\right] \text { for all } t \geq 0, f \in \mathrm{UC}_{\kappa} \text { and } x \in \mathbb{R}^{d} .
$$

Lemma 6.8 For every $t \geq 0, \lambda \in \Lambda, f \in \mathrm{UC}_{\kappa}$, and $x \in \mathbb{R}^{d}$,

$\mathbb{E}\left[1+\left|x+\sigma W_{t}+\mu t\right|^{2}\right] \kappa(x) \leq e^{\omega t} \quad$ and $\quad \mathbb{E}\left[\left|f\left(x+\sigma W_{t}+\mu t\right)\right|\right] \leq\|f\|_{\kappa}\left(1+|x|^{2}\right) e^{\omega t}$, where $\omega:=\sup _{\lambda \in \Lambda} \max \left\{1+|\mu|^{2}+\left|\sigma^{2}\right|, \sqrt{2}|\mu|\right\}<\infty$. 
Proof We use Young's inequality to estimate

$$
\begin{aligned}
& \mathbb{E}\left[1+\left|x+\sigma W_{t}+\mu t\right|^{2}\right] \kappa(x) \\
& =\mathbb{E}\left[1+|x|^{2}+2\left\langle x, \sigma W_{t}\right\rangle+2\langle x, \mu t\rangle+\left|\sigma W_{t}\right|^{2}+2\left\langle\sigma W_{t}, \mu t\right\rangle+|\mu|^{2} t^{2}\right] \kappa(x) \\
& \leq\left(1+|x|^{2}+2|x| \cdot|\mu| t+|\sigma|^{2} t+|\mu|^{2} t^{2}\right) \kappa(x) \\
& \leq\left(1+|x|^{2}+|x|^{2} t+|\mu|^{2} t+|\sigma|^{2} t+|\mu|^{2} t^{2}\right) \kappa(x) \\
& \leq\left(1+\left(1+|\mu|^{2}+|\sigma|^{2}\right) t+|\mu|^{2} t^{2}\right) \leq e^{\omega t} .
\end{aligned}
$$

Here, the last inequality holds, because $1+a t+b t^{2} \leq e^{\max \{a, \sqrt{2 b}\} t}$ for all $a, b, t \geq 0$. The second inequality of the claim follows from the first one, see the proof of Lemma 6.5 .

Lemma 6.8 ensures that $S_{\lambda}(t) f: \mathbb{R}^{d} \rightarrow \mathbb{R}$ and $I(t): \mathbb{R}^{d} \rightarrow \mathbb{R}$ are well-defined functions for all $\lambda \in \Lambda, t \geq 0$ and $f \in \mathrm{UC}_{\kappa}$. We denote by $\operatorname{tr}(a)$ the trace of a matrix $a \in \mathbb{R}^{d \times d}$. Recall that $\nabla_{\mu} f:=\langle\mu, \nabla f\rangle$.

Theorem 6.9 The family $(I(t))_{t \geq 0}$ satisfies Assumptions 2.1 and 2.4, i.e., Theorem 2.5 yields a semigroup $(S(t))_{t \geq 0}$ on $\mathrm{UC}_{\kappa}$ associated to $(I(t))_{t \geq 0}$. Furthermore, it holds $C_{c}^{2} \subset$ $D(A)$ with

$$
A f=\sup _{\lambda \in \Lambda}\left(\frac{1}{2} \operatorname{tr}\left(\sigma^{2} D^{2} f\right)+\nabla_{\mu} f\right) \quad \text { for all } f \in C_{c}^{2} .
$$

Proof First, we show that $(I(t))_{t \geq 0}$ satisfies Assumptions 2.1 and 2.4. Since $I(t)$ is translation-invariant and a contraction w.r.t. the supremum norm, we conclude $I(t): \operatorname{Lip}_{b}(c) \rightarrow \operatorname{Lip}_{b}(c)$ for all $c, t \geq 0$. Furthermore, it follows from Lemma 6.8 that $\|I(t) f\|_{\kappa} \leq e^{\omega t}\|f\|_{\kappa}$ and $\|I(t) f-I(t) g\|_{\kappa} \leq e^{\omega t}\|f-g\|_{\kappa}$ for all $t \geq 0$ and $f, g \in \mathrm{UC}_{\kappa}$. In particular, we have $I(t): \mathrm{UC}_{\kappa} \rightarrow \mathrm{UC}_{\kappa}$ for all $t \geq 0$. In addition, for every $f \in C_{c}^{2}$, Itô's formula implies

$$
\|I(t) f-f\|_{\kappa} \leq c t \quad \text { for all } t \geq 0,
$$

where $c:=\sup _{i n \Lambda}\left(|\mu| \cdot\|\nabla f\|_{\infty}+\frac{1}{2}|\sigma|^{2}\left\|D^{2} f\right\|_{\infty}\right)<\infty$.

Second, we show that

$$
\lim _{t \downarrow 0}\left\|\frac{I(t) f-f}{t}-\sup _{\lambda \in \Lambda}\left(\frac{1}{2} \operatorname{tr}\left(\sigma^{2} D^{2} f\right)+\nabla_{\mu} f\right)\right\|_{\infty}=0 \quad \text { for all } f \in C_{c}^{2} .
$$

Let $f \in C_{c}^{2}$. We use Itô's formula to estimate

$$
\begin{aligned}
& \left.\left\|\frac{I(t) f-f}{t}-\sup _{\lambda \in \Lambda}\left(\frac{1}{2} \operatorname{tr}\left(\sigma^{2} D^{2} f\right)+\nabla_{\mu} f\right)\right\|_{\infty} \leq \sup _{\lambda \in \Lambda} \| \frac{S_{\lambda}(t) f-f}{t}-\frac{\operatorname{tr}\left(\sigma^{2} D^{2} f\right.}{2}\right)-\nabla_{\mu} f \|_{\infty} \\
& \leq \sup _{\lambda \in \Lambda} \frac{1}{t} \int_{0}^{t}\left(\left\|\nabla_{\mu} f\left(\cdot+X_{s}^{\lambda}\right)-\nabla_{\mu} f\right\|_{\infty}+\frac{1}{2}\left\|\operatorname{tr}\left(\sigma^{2} D^{2} f\right)\left(\cdot+X_{s}^{\lambda}\right)-\operatorname{tr}\left(\sigma^{2} D^{2} f\right)\right\|_{\infty}\right) \mathrm{d} s,
\end{aligned}
$$

where $X_{s}^{\lambda}:=\sigma W_{t}+\mu s$ for all $\lambda \in \mathbb{R}^{d}$ and $s \geq 0$. Since $\Lambda$ is bounded and $f \in C_{c}^{2}$, the right hand side converges to zero as $t \downarrow 0$. It follows from $\|\cdot\|_{\kappa} \leq\|\cdot\|_{\infty}$, Theorem 4.3 and Lemma 4.4 that $C_{c}^{2} \subset D(A)$ with

$$
A f=\sup _{\lambda \in \Lambda}\left(\frac{1}{2} \operatorname{tr}\left(\sigma^{2} D^{2} f\right)+\nabla_{\mu} f\right) \text { for all } f \in C_{c}^{2} .
$$




\subsection{Ordinary Differential Equations}

In this subsection, we obtain the well-known existence and uniqueness result for ODEs with locally Lipschitz continuous data. Let $f: \mathbb{R}^{d} \rightarrow \mathbb{R}^{d}$ be a function, which satisfies the following conditions:

- There exists $K \geq 0$ such that $|f(x)| \leq K(1+|x|)$ for all $x \in \mathbb{R}^{d}$.

- For every $r \geq 0$ there exists $L_{r} \geq 0$ such that

$$
|f(x)-f(y)| \leq L_{r}|x-y| \text { for all } x, y \in B(0, r) .
$$

We define $I(t) x:=x+t f(x)$ for all $t \geq 0$ and $x \in \mathbb{R}^{d}$.

Theorem 6.10 The family $(I(t))_{t \geq 0}$ satisfies Assumptions 2.1 and 2.4, i.e., Theorem 2.5 yields a semigroup $(S(t))_{t \geq 0}$ on $\mathbb{R}^{d}$ associated to $(I(t))_{t \geq 0}$. Furthermore, for every $x \in \mathbb{R}^{d}$ the Cauchy problem

$$
y^{\prime}(t)=f(y(t)) \quad \text { for all } t \geq 0, \quad y(0)=x,
$$

has a unique classical solution given by $y(t):=S(t) x$.

Proof First, we verify Assumptions 2.1 and 2.4. It holds $|I(t) x| \leq \alpha(r, t)$ for all $r, t \geq 0$ and $x \in \mathbb{R}^{d}$, where

$$
\alpha(r, t):= \begin{cases}e^{2 K t}, & r \leq 1, \\ e^{2 K t} r, & r>1 .\end{cases}
$$

Moreover, we have $|I(t) x-I(t) y| \leq e^{L_{r} t}|x-y|$ for all $r, t \geq 0$ and $x, y \in B(0, r)$. In addition, Lemma 2.7 implies that the sequence $\left(I\left(\pi_{n}^{t}\right) x\right)_{n \in \mathbb{N}} \subset \mathbb{R}^{d}$ is bounded, and therefore relatively compact for all $(x, t) \in \mathbb{R}^{d} \times \mathcal{T}$. Theorem 2.5 yields a semigroup $(S(t))_{t \geq 0}$ on $\mathbb{R}^{d}$ associated to $(I(t))_{t \geq 0}$.

Second, we note that

$$
\frac{I(t) x-x}{t}-f(x)=0 \quad \text { for all } t>0 \text { and } x \in \mathbb{R}^{d} .
$$

Furthermore, one can show that condition (4.2) holds for all $x, y \in \mathbb{R}^{d}$, for details we refer to the proofs of Lemma 6.13 and Theorem 6.14 below. Hence, it follows from Theorem 4.3 that $D(A)=\mathbb{R}^{d}$ and $A x=f(x)$ for all $x \in \mathbb{R}^{d}$. Let $x \in \mathbb{R}^{d}$ and define $y(t):=S(t) x$ for all $t \geq 0$. For every $t \geq 0$, the right-derivative of $y$ exists and is given by $A y(t)=f(y(t))$. Since $y$ and $f(y(\cdot))$ are continuous, it follows from [28, Corollary 1.2 in Section 2] that $y$ is continuously differentiable. The uniqueness follows from Theorem 4.8.

\subsection{Lipschitz Perturbation}

Throughout this subsection, we consider vector valued functions $f: \mathbb{R}^{d} \rightarrow \mathbb{R}^{m}$. We construct a semigroup corresponding to a perturbed linear semigroup. The nonlinear generator is the sum of the linear generator and a nonlinear zero-order coupling. The ODEs from the previous section are including in this setting, as well as reaction-diffusion equations, see Example 6.15. We endow $\mathbb{R}^{m}$ with the order $x \leq y$ if and only if $x_{i} \leq y_{i}$ for $i=1, \ldots, m$. 
Assumption 6.11 Let $\left(S_{0}(t)\right)_{t \geq 0}$ be a strongly continuous monotone linear semigroup on $C_{0}\left(\mathbb{R}^{d} ; \mathbb{R}^{m}\right)$, which satisfies the following condition:

1. There exists $\omega \geq 0$ such that $\left\|S_{0}(t) f\right\|_{\infty} \leq e^{\omega t}\|f\|_{\infty}$ for all $t \geq 0$ and $f \in C_{0}$.

2. $\mathcal{L}^{S_{0}} \cap \operatorname{Lip}_{0} \subset C_{0}$ is dense.

3. $S_{0}(t): \operatorname{Lip}_{0}(c) \rightarrow \operatorname{Lip}_{0}\left(e^{\omega t} c\right)$ for all $c, t \geq 0$.

4. $\left.\lim _{|x| \rightarrow \infty} \sup _{t \in[0, T]} \mid S_{0}(t) f\right)(x) \mid=0$ for all $T \geq 0$ and $f \in C_{0}$.

Furthermore, let $\Psi: \mathbb{R}^{m} \rightarrow \mathbb{R}^{m}$ be a continuous function with $\Psi(0)=0$, which satisfies the following conditions:

5. There exists $K \geq 0$ such that $|\Psi(x)| \leq K(1+|x|)$ for all $x \in \mathbb{R}^{m}$.

6. For every $r \geq 0$ there exists $L_{r} \geq 0$ such that

$$
|\Psi(x)-\Psi(y)| \leq L_{r}|x-y| \text { for all } x, y \in B(0, r) .
$$

W.l.o.g. we assume that the mapping $r \mapsto L_{r}$ is non-decreasing. We define

$$
(I(t) f)(x):=\left(S_{0}(t) f\right)(x)+t \Psi(f(x)) \text { for all } t \geq 0, f \in C_{0} \text { and } x \in \mathbb{R}^{d} .
$$

Theorem 6.12 The family $(I(t))_{t \geq 0}$ satisfies Assumptions 2.1 and 2.4, i.e., Theorem 2.5 yields a semigroup $(S(t))_{t \geq 0}$ on $C_{0}$ associated to $(I(t))_{t \geq 0}$.

Proof First, we verify Assumption 2.1. It holds $I(t): C_{0} \rightarrow C_{0}$, because $S_{0}(t): C_{0} \rightarrow C_{0}$ and $\Psi$ is a continuous function with $\Psi(0)=0$. Clearly, it holds $I(0)=\operatorname{id}_{C_{0}}$. Let $t \geq 0$ and $f \in C_{0}$. If $\|f\|_{\infty} \leq 1$, we use Assumption 6.11(i) and (v) to estimate

$$
\|I(t) f\|_{\infty} \leq e^{\omega t}\|f\|_{\infty}+t K\left(1+\|f\|_{\infty}\right) \leq e^{\omega t}+2 K t \leq e^{(\omega+2 K) t} .
$$

Similarly, if $\|f\|_{\infty}>1$, we obtain the estimate $\|I(t) f\|_{\infty} \leq e^{(\omega+2 K) t}\|f\|_{\infty}$. Hence, Assumption 2.1(i) holds with

$$
\alpha(r, t):=\left\{\begin{array}{ll}
e^{(\omega+2 K) t}, & r \leq 1, \\
e^{(\omega+2 K) t} r, & r>1,
\end{array} \quad \text { for all } r, t \geq 0 .\right.
$$

Furthermore, for every $r, t \geq 0$ and $f, g \in B(0, R)$, Assumption 6.11(i) and (vi) implies

$$
\|I(t) f-I(t) g\|_{\infty} \leq e^{\omega t}\|f-g\|_{\infty}+t L_{r}\|f-g\|_{\infty} \leq e^{\left(\omega+L_{r}\right) t}\|f-g\|_{\infty} .
$$

Second, we show that $\mathcal{L}^{I}=\mathcal{L}^{S_{0}}$. For every $t \geq 0$ and $f \in C_{0}$, it follows from $\Psi(0)=0$ and Assumption 6.11(vi) that

$$
\left\|I(t) f-S_{0}(t) f\right\|_{\infty}=t\|\psi(f)\|_{\infty} \leq t L_{r}, \quad \text { where } \quad r:=\|f\|_{\infty} .
$$

In particular, Assumption 6.11(ii) implies that $\mathcal{L}^{I} \cap \operatorname{Lip}_{0} \subset C_{0}$ is dense. Hence, in order to verify Assumption 2.4, it suffices to show that the assumptions from Lemma 3.1 are satisfied for all $f \in \operatorname{Lip}_{0}$.

Third, we show that

$$
I(t): \operatorname{Lip}_{0}(c) \cap B(0, r) \rightarrow \operatorname{Lip}_{0}\left(e^{\left(\omega+L_{r}\right) t} c\right) \text { for all } c, r, t \geq 0 .
$$

For every $c, r, t \geq 0, f \in \operatorname{Lip}_{0}(c) \cap B(0, r)$ and $x, y \in \mathbb{R}^{d}$, it follows from Assumption 6.11(iii) and (v) that

$$
\begin{aligned}
|(I(t) f)(x)-(I(t) f)(y)| & \leq\left|\left(S_{0}(t) f\right)(x)-\left(S_{0}(t) f\right)(y)\right|+t|\Psi(f(x))-\Psi(f(y))| \\
& \leq\left(e^{\omega t}+L_{r} t\right) c|x-y| \leq e^{\left(\omega+L_{r}\right) t} c|x-y| .
\end{aligned}
$$


Let $c \geq 0, f \in \operatorname{Lip}_{0}(c)$ and $t \in \mathcal{T}$. By induction, it follows that $I\left(\pi_{n}^{t}\right) f \in \operatorname{Lip}_{0}\left(e^{\left(\omega+L_{r}\right) t} c\right)$ for all $n \in \mathbb{N}$, where $r:=\alpha(c, t)$. In particular, the sequence $I\left(\pi_{n}^{t}\right)_{n \in \mathbb{N}}$ is equicontinuous.

Fourth, it remains to show that

$$
\lim _{|x| \rightarrow \infty} \sup _{n \in \mathbb{N}}\left|\left(I\left(\pi_{n}^{t}\right) f\right)(x)\right|=0 \quad \text { for all } f \in \operatorname{Lip}_{0} \text { and } t \in \mathcal{T} .
$$

To do so, for every $r, t \geq 0$, we define

$$
J_{r}(t): C_{0} \rightarrow C_{0}, f \mapsto S_{0}(t) f+t L_{r} f .
$$

Since $S_{0}(t)$ is monotone, it holds $J_{r}(t) f \leq J_{r}(t) g$ for all $r, t \geq 0$ and $f, g \in C_{0}$ with $0 \leq f \leq g$. Moreover, the mapping $r \mapsto J_{r}(t) f$ is non-decreasing for all $f \in C_{0}$ with $f \geq 0$, because we assumed the mapping $r \mapsto L_{r}$ to be non-decreasing. We show by induction that

$$
\left|I(t)^{k} f\right| \leq\left(J_{\alpha(r, k t)}(t)\right)^{k}|f| \quad \text { for all } k \in \mathbb{N}, r, t \geq 0 \text { and } f \in B(0, r) .
$$

Let $r, t \geq 0$ and $f \in B(0, r)$. We use the monotonicity of $S_{0}(t), \Psi(0)=0$ and Assumption 6.11(vi) to estimate

$$
|I(t) f| \leq\left|S_{0}(t) f\right|+t|\Psi(f)| \leq S_{0}(t)|f|+L_{r} t|f|=J_{r}(t)|f| \leq J_{\alpha(r, t)}(t)|f| .
$$

For the induction step, we assume that inequality (6.12) holds for some fixed $k \in \mathbb{N}$. Let $r, t \geq 0$ and $f \in B(0, r)$. It follows from $I(t) f \in B(0, \alpha(r, t))$, inequality (6.12), inequality (2.1) and the monotonicity of $J$ that

$$
\begin{aligned}
\left|I(t)^{k+1} f\right| & =\left|I(t)^{k} I(t) f\right| \leq\left(J_{\alpha(\alpha(r, t), k t)}(t)\right)^{k}|I(t) f| \\
& \leq\left(J_{\alpha(r,(k+1) t)}(t)\right)^{k} J_{r}(t)|f| \leq\left(J_{\alpha(r,(k+1) t)}(t)\right)^{k+1}|f| .
\end{aligned}
$$

Fifth, since $\left(S_{0}(t)\right)_{t \geq 0}$ is a linear semigroup, the binomial theorem implies

$$
J_{r}(t)^{k}=\sum_{l=0}^{k}\left(\begin{array}{l}
k \\
l
\end{array}\right)\left(L_{r} t\right)^{l} S_{0}((k-l) t) \quad \text { for all } r, t \geq 0 \text { and } k \in \mathbb{N} .
$$

Let $f \in C_{0}, t \in \mathcal{T}$ and $\varepsilon>0$. Choose $n_{0} \in \mathbb{N}$ with $2^{n} t \in \mathbb{N}$ for all $n \geq n_{0}$. By inequality (6.12), it holds

$$
\left|I\left(\pi_{n}^{t}\right) f\right| \leq J_{r}\left(2^{-n}\right)^{2^{n} t}|f| \text { for all } n \geq n_{0},
$$

where $r:=\alpha\left(\|f\|_{\infty}, t\right)$. In addition, by Assumption 6.11(iv) there exists $R \geq 0$ with

$$
\left|\left(S_{0}(s)|f|\right)(x)\right| \leq e^{-L_{r} t} \varepsilon \quad \text { for all } s \in[0, t] \text { and } x \in B(0, R)^{c} .
$$

Let $x \in B(0, R)^{c}, n \geq n_{0}$ and $k:=2^{n} t$. We use Eqs. (6.13)-(6.15) and the binomial theorem to estimate

$$
\begin{aligned}
& \left|I\left(\pi_{n}^{t}\right) f\right|(x) \leq\left(J_{r}\left(2^{-n}\right)^{k}|f|\right)(x)=\sum_{l=0}^{k}\left(\begin{array}{l}
k \\
l
\end{array}\right)\left(L_{r} 2^{-n}\right)^{l}\left(S_{0}\left((k-l) 2^{-n}\right)|f|\right)(x) \\
& \leq e^{-L_{r} t} \varepsilon \sum_{l=0}^{k}\left(\begin{array}{l}
k \\
l
\end{array}\right)\left(L_{r} 2^{-n}\right)^{l}=e^{-L_{r} t} \varepsilon\left(1+L_{r} 2^{-n}\right)^{k} \leq e^{-L_{r} t} \varepsilon e^{L_{r} k 2^{-n}}=\varepsilon .
\end{aligned}
$$

We obtain $\lim _{|x| \rightarrow \infty} \sup _{n \in \mathbb{N}}\left|\left(I\left(\pi_{n}^{t}\right) f\right)(x)\right|=0$ for all $(f, t) \in C_{0} \times \mathcal{T}$.

To determine the generator of $(S(t))_{t \geq 0}$, we need the following recursion. 
Lemma 6.13 For every $f, g \in C_{0}$ and $k, n \in \mathbb{N}$,

$$
\begin{aligned}
& I\left(2^{-n}\right)^{k} f-I\left(2^{-n}\right)^{k} g \\
& =S_{0}\left(k 2^{-n}\right)(f-g)+2^{-n} \sum_{l=0}^{k-1} S_{0}\left((k-1-l) 2^{-n}\right)\left(\Psi\left(I\left(2^{-n}\right)^{l} f\right)-\Psi\left(I\left(2^{-n}\right)^{l} g\right)\right) .
\end{aligned}
$$

Proof Let $f, g \in C_{0}$ and $n \in \mathbb{N}$. We prove the claim by induction w.r.t. $k \in \mathbb{N}$. For $k=1$, linearity of $S_{0}\left(2^{-n}\right)$ implies

$$
I\left(2^{-n}\right) f-I\left(2^{-n}\right) g=S_{0}\left(2^{-n}\right)(f-g)+2^{-n}(\Psi(f)-\Psi(g)) .
$$

For the induction step, we assume that the claim holds for some fixed $k \in \mathbb{N}$. Since $\left(S_{0}(t)\right)_{t \geq 0}$ is a linear semigroup, we obtain

$$
\begin{aligned}
& I\left(2^{-n}\right)^{k+1} f-I\left(2^{-n}\right)^{k+1} g=I\left(2^{-n}\right)^{k} I\left(2^{-n}\right) f-I\left(2^{-n}\right)^{k} I\left(2^{-n}\right) g \\
& =S_{0}\left(k 2^{-n}\right)\left(I\left(2^{-n}\right) f-I\left(2^{-n}\right) g\right) \\
& \quad+2^{-n} \sum_{l=0}^{k-1} S_{0}\left((k-1-l) 2^{-n}\right)\left(\Psi\left(I\left(2^{-n}\right)^{l} I\left(2^{-n}\right) f\right)-\Psi\left(I\left(2^{-n}\right)^{l} I\left(2^{-n}\right) g\right)\right) \\
& =S_{0}\left(k 2^{-n}\right) S_{0}\left(2^{-n}\right)(f-g)+2^{-n} S_{0}\left(k 2^{-n}\right)(\Psi(f)-\Psi(g)) \\
& \quad+2^{-n} \sum_{l=0}^{k-1} S_{0}\left((k-(l+1)) 2^{-n}\right)\left(\Psi\left(I\left(2^{-n}\right)^{l+1} f\right)-\Psi\left(I\left(2^{-n}\right)^{l+1} g\right)\right) \\
& =S_{0}\left((k+1) 2^{-n}\right)(f-g)+2^{-n} S_{0}\left(k 2^{-n}\right)(\Psi(f)-\Psi(g)) \\
& \quad+2^{-n} \sum_{l=1}^{k} S_{0}\left((k-l) 2^{-n}\right)\left(\Psi\left(I\left(2^{-n}\right)^{l} f\right)-\Psi\left(I\left(2^{-n}\right)^{l} g\right)\right) \\
& =S_{0}\left((k+1) 2^{-n}\right)(f-g)+2^{-n} \sum_{l=0}^{k} S_{0}\left((k-l) 2^{-n}\right)\left(\Psi\left(I\left(2^{-n}\right)^{l} f\right)-\Psi\left(I\left(2^{-n}\right)^{l} g\right)\right) .
\end{aligned}
$$

Theorem 6.14 It holds $D\left(A_{0}\right) \subset D(A)$ with $A f=A_{0} f+\psi(f)$ for all $f \in D\left(A_{0}\right)$, where $A_{0}$ denotes the generator of $\left(S_{0}(t)\right)_{t \geq 0}$.

Proof For every $f \in D\left(A_{0}\right)$,

$$
\frac{I(t) f-f}{t}-A_{0}-\Psi(f)=\frac{S_{0}(t) f-f}{t}-A_{0} \rightarrow 0 \quad \text { as } t \downarrow 0 .
$$

It remains to show inequality (4.2). Let $f, g \in C_{0}$ and $\varepsilon>0$. Let $r:=\max \left\{\|f\|_{\infty},\|g\|_{\infty}\right\}$ and $c:=\alpha(2 r, t)$. Since $\left(S_{0}(t)\right)_{t \geq 0}$ is strongly continuous, there exists $t_{0} \in(0,1]$ such that

$$
\left\|S_{0}(t) g-g\right\|_{\infty} \leq \frac{\varepsilon}{2} \quad \text { and } \quad e^{\left(2 \omega+L_{c}\right) t} L_{c} t\|g\|_{\infty} \leq \frac{\varepsilon}{2} \quad \text { for all } t \in\left[0, t_{0}\right] .
$$


Let $k, n \in \mathbb{N}$ with $k 2^{-n} \leq t_{0}$. We use Lemma 6.13, Assumption 6.11(i) and (vi), and Lemma 2.7 to estimate

$$
\begin{aligned}
& \left\|\frac{I\left(2^{-n}\right)^{k}\left(f+2^{-n} g\right)-I\left(2^{-n}\right)^{k} f}{2^{-n}}-g\right\|_{\infty} \\
& \leq\left\|S_{0}\left(k 2^{-n}\right) g-g\right\|_{\infty} \\
& \quad+\sum_{l=0}^{k-1}\left\|S_{0}\left((k-1-l) 2^{-n}\right)\left(\Psi\left(I\left(2^{-n}\right)^{l}\left(f+2^{-n} g\right)\right)-\Psi\left(I\left(2^{-n}\right)^{l} f\right)\right)\right\|_{\infty} \\
& \leq \frac{\varepsilon}{2}+\sum_{l=0}^{k-1} e^{\omega(k-1-l) 2^{-n}} L_{c} e^{\left(\omega+L_{c}\right) l 2^{-n}}\left\|2^{-n} g\right\|_{\infty} \\
& \leq \frac{\varepsilon}{2}+k 2^{-n} e^{\omega(k-1) 2^{-n}} L_{c} e^{\left(\omega+L_{c}\right) k 2^{-n}}\|g\|_{\infty} \leq \varepsilon .
\end{aligned}
$$

Example 6.15 Let $\left(W_{t}\right)_{t \geq 0}$ be a $d$-dimensional Brownian motion on a probability space $(\Omega, \mathcal{F}, \mathbb{P})$. We define

$$
\left(S_{0}(t) f\right)(x):=\mathbb{E}\left[f\left(x+W_{t}\right)\right] \text { for all } t \geq 0, f \in C_{0} \text { and } x \in \mathbb{R}^{d},
$$

Then, the family $\left(S_{0}(t)\right)_{t \geq 0}$ satisfies Assumption 6.11, and

$$
\lim _{t \downarrow 0}\left\|\frac{S_{0}(t) f-f}{t}-\frac{1}{2} \Delta f\right\|_{\infty}=0 \quad \text { for all } f \in C_{0}^{2},
$$

where the Laplacian is defined component-wise. Furthermore, let $\Psi$ be a function, which satisfies Assumption 6.11, and $(S(t))_{t \geq 0}$ an associated semigroup as in Theorem 6.12. Then, by Theorem 6.14, it holds $C_{0}^{2} \subset D(A)$ with $A f=\frac{1}{2} \Delta f+\Psi(f)$ for all $f \in C_{0}^{2}$. Equations of the form $\partial_{t} f=\frac{1}{2} \Delta f+\Psi(f)$ are known as reaction diffusion systems, see, e.g. [23, Section 7.3].

Funding Open Access funding enabled and organized by Projekt DEAL.

Open Access This article is licensed under a Creative Commons Attribution 4.0 International License, which permits use, sharing, adaptation, distribution and reproduction in any medium or format, as long as you give appropriate credit to the original author(s) and the source, provide a link to the Creative Commons licence, and indicate if changes were made. The images or other third party material in this article are included in the article's Creative Commons licence, unless indicated otherwise in a credit line to the material. If material is not included in the article's Creative Commons licence and your intended use is not permitted by statutory regulation or exceeds the permitted use, you will need to obtain permission directly from the copyright holder. To view a copy of this licence, visit http://creativecommons.org/licenses/by/4.0/.

\section{References}

1. Barbu, V.: Nonlinear Differential Equations of Monotone Types in Banach Spaces Springer Monographs in Mathematics. Springer, New York (2010)

2. Bénilan, P., Crandall, M.G.: Completely Accretive Operators. In: Semigroup Theory and Evolution Equations (Delft, 1989), Volume 135 of Lecture Notes in Pure and Appl. Math., pp. 41-75. Dekker, New York (1991)

3. Blessing, J., Kupper, M.: Viscous hamilton-Jacobi equations in exponential Orlicz hearts. arXiv:2104.06433 (2021)

4. Brézis, H.: Monotonicity methods in Hilbert spaces and some applications to nonlinear partial differential equations. In: Contributions to Nonlinear Functional Analysis (Proc. Sympos., Math. Res. Center, Univ. Wisconsin, Madison, Wis., 1971), pp. 101-156. Academic Press, New York (1971) 
5. Butko, Y.A.: The method of Chernoff approximation. In: Conference on Semigroups of Operators: Theory and Applications, pp. 19-46. Springer (2018)

6. Butko, Y.A., Smolyanov, O.G., Shilling, R.L.: Feynman formulas for Feller semigroups. Dokl. Akad. Nauk 434(1), 7-11 (2010)

7. Chernoff, P.R.: Note on product formulas for operator semigroups. J. Funct Anal. 2, 238-242 (1968)

8. Chernoff, P.R.: Product formulas, nonlinear semigroups, and addition of unbounded operators, volume 140 American Mathematical Soc. (1974)

9. Coquet, F., Hu, Y., Mémin, J., Peng, S.: Filtration-consistent nonlinear expectations and related $g$-expectations. Probab. Theory Related Fields 123(1), 1-27 (2002)

10. Crandall, M.G., Ishii, H., Lions, P.-L.: User's guide to viscosity solutions of second order partial differential equations. Bull. Amer. Math Soc. (N.S.) 27(1), 1-67 (1992)

11. Delbaen, F., Hu, Y., Bao, X.: Backward SDEs with superquadratic growth. Probab. Theory Relat. Fields 150(1-2), 145-192 (2011)

12. Denk, R., Kupper, M., Nendel, M.: A semigroup approach to nonlinear Lévy processes. Stochastic Process Appl. 130, 1616-1642 (2020)

13. Denk, R., Kupper, M., Nendel, M.: Convex semigroups on lattices of continuous functions. Forthcoming Publ. Res. Inst. Math. Sci (2021)

14. Denk, R., Kupper, M., Nendel, M.: Convex semigroups on $L^{p}$-like spaces. J. Evol. Equ. 21(2), 24912521 (2021)

15. El Karoui, N., Peng, S., Quenez, M.C.: Backward stochastic differential equations in finance. Math. Financ. 7(1), 1-71 (1997)

16. Evans, L.C.: Nonlinear Semigroup Theory and Viscosity Solutions of Hamilton-Jacobi PDE. In: Nonlinear Semigroups, Partial Differential Equations and Attractors (Washington, D.C., 1985), volume 1248 of Lecture Notes in Math, pp. 63-77. Springer, Berlin (1987)

17. Gomilko, A., Kosowicz, S., Tomilov, Y.: A general approach to approximation theory of operator semigroups. J. Math. Pures Appl. 127(9), 216-267 (2019)

18. Hollender, J.: Lévy-Type Processes under Uncertainty and Related Nonlocal Equations, Phd thesis TU Dresden (2016)

19. Hu, M., Peng, S.: $g$-Lévy processes under sublinear expectations. Preprint (2009)

20. Kato, T.: Nonlinear semigroups and evolution equations. J. Math. Soc. Japan 19, 508-520 (1967)

21. Kazi-Tani, N., Possamaï D., Zhou, C., et al.: Second-order bsdes with jumps: formulation and uniqueness. Ann. Appl. Probab. 25(5), 2867-2908 (2015)

22. Kühn, F.: Viscosity solutions to Hamilton-Jacobi-Bellman equations associated with sublinear Lévy(type) processes. ALEA Lat. Am. J. Probab. Math. Stat., (16):531-559 (2019)

23. Lunardi, A.: Analytic semigroups and optimal regularity in parabolic problems. Modern Birkhäuser Classics. Birkhäuser/Springer, Basel (1995). [2013 reprint of the 1995 original] [MR1329547]

24. Nendel, M., Röckner, M.: Upper envelopes of families of Feller semigroups and viscosity solutions to a class of nonlinear Cauchy problems. Forthcoming in SIAM J. Control Optim (2021)

25. Neufeld, A., Nutz, M.: Nonlinear lévy processes and their characteristics. Trans. Amer. Math. Soc. 369(1), 69-95 (2017)

26. Nisio, M.: On a non-linear semi-group attached to stochastic optimal control. Publ. Res. Inst. Math. Sci. 12(2), 513-537 (1976/77)

27. Orlov, Y.N., Sakbaev, V.Z., Smolyanov, O.G.: Feynman formulas for nonlinear evolution equations. Dokl. Akad Nauk 477(3), 271-275 (2017)

28. Pazy, A.: Semigroups of linear operators and applications to partial differential equations, Volume 44 of Applied Mathematical Sciences. Springer, New York (1983)

29. Peng, S.: $G$-expectation, $G$-Brownian Motion and Related Stochastic Calculus of Itô Type. In: Stochastic Analysis and Applications, Volume 2 of Abel Symp, pp. 541-567. Springer, Berlin (2007)

30. Peng, S.: Multi-dimensional $G$-Brownian motion and related stochastic calculus under $G$-expectation. Stochastic Process Appl. 118(12), 2223-2253 (2008)

31. Smolyanov, O.G., Tokarev, A.G., Truman, A.: Hamiltonian Feynman path integrals via the Chernoff formula. J. Math Phys. 43(10), 5161-5171 (2002)

32. Soner, H.M., Touzi, N., Zhang, J.: Wellposedness of second order backward SDEs. Probab. Theory Relat. Fields 153(1-2), 149-190 (2012)

33. Trotter, H.F.: Approximation of semi-groups of operators. Pacific J. Math. 8, 887-919 (1958)

34. Trotter, H.F.: On the product of semi-groups of operators. Proc. Amer. Math. Soc. 10, 545-551 (1959)

35. Wnuk, W.: Banach Lattices with Order Continuous Norms. Advanced topics in mathematics Polish Scientific Publishers PWN (1999)

Publisher's Note Springer Nature remains neutral with regard to jurisdictional claims in published maps and institutional affiliations. 